\title{
WestVirginiaUniversity
}

THE RESEARCH REPOSITORY @ WVU

Graduate Theses, Dissertations, and Problem Reports

2013

\section{A Study of Exploiting Objectness for Robust Online Object Tracking}

Raghu Kiran Yalamanchili

West Virginia University

Follow this and additional works at: https://researchrepository.wvu.edu/etd

\section{Recommended Citation}

Yalamanchili, Raghu Kiran, "A Study of Exploiting Objectness for Robust Online Object Tracking" (2013). Graduate Theses, Dissertations, and Problem Reports. 3625.

https://researchrepository.wvu.edu/etd/3625

This Thesis is protected by copyright and/or related rights. It has been brought to you by the The Research Repository @ WVU with permission from the rights-holder(s). You are free to use this Thesis in any way that is permitted by the copyright and related rights legislation that applies to your use. For other uses you must obtain permission from the rights-holder(s) directly, unless additional rights are indicated by a Creative Commons license in the record and/ or on the work itself. This Thesis has been accepted for inclusion in WVU Graduate Theses, Dissertations, and Problem Reports collection by an authorized administrator of The Research Repository @ WVU. For more information, please contact researchrepository@mail.wvu.edu. 


\title{
A Study of Exploiting Objectness for Robust Online Object Tracking
}

\author{
by \\ Raghu Kiran Yalamanchili \\ Thesis submitted to the \\ Benjamin M. Statler College of Engineering and Mineral Resources \\ at West Virginia University \\ in partial fulfillment of the requirements \\ for the degree of \\ Master of Science \\ in \\ Electrical Engineering \\ Lawrence A. Hornak, Ph. D. \\ Xin Li, Ph. D., Chair \\ Arun A. Ross, Ph. D. \\ Lane Department of Computer Science and Electrical Engineering
}

Morgantown, West Virginia

2013

Keywords: Object tracking, condensation, objectness, online learning, adaptive appearance model

Copyright (C) 2013 Raghu Kiran Yalamanchili 


\author{
Abstract \\ A study of exploiting objectness for robust online object tracking \\ by \\ Raghu Kiran Yalamanchili \\ Master of Science in Electrical Engineering \\ West Virginia University \\ Xin Li, Ph. D., Chair
}

Tracking is a fundamental problem in many computer vision applications. Despite the progress over the last decade, there still exist many challenges especially when the problem is posed in real world scenarios (e.g., cluttered background, occluded objects). Among them drifting has been widely observed to be a problem common to the class of online tracking algorithms - i.e., when challenges such as occlusion or nonlinear deformation of the object occurs, the tracker might lose the target completely in subsequent frames in an image sequence. In this work, we propose to exploit the objectness to partially alleviate the drifting problem with the class of online object tracking and verify the effectiveness of this idea by extensive experimental results. More specifically, a recently developed objectness measure was incorporated into Incremental Learning for Visual Tracking (IVT) algorithm in a principled way. We have come up with a strategy of reinitializing the training samples in the proposed approach to improve the robustness of online tracking. Experimental results show that using objectness measure does help to alleviate its drift to background for certain challenging sequences. 
To My Parents and Manasa 


\section{Acknowledgements}

I feel very fortunate to have an opportunity to work with $\mathrm{Dr}$. Li. He has been very patient and supportive all throughout my graduate studies. The discussions I had with him helped to shape my perspective about a research problem and enhanced my problem solving skills. He has always been inspirational and motivated me to work and think harder. I am indebted for all his help and I consider him as a true mentor for my life.

I would like to offer my sincere thanks to Dr. Arun Ross for his valuable suggestions towards this thesis. He is a great teacher and his courses has helped immensely to increase my knowledge in the fields of biometrics and pattern recognition. He has always been patient in answering all my questions after the classes and offered valuable suggestions.

I thank Dr. Lawrence Hornak for serving on the committee. He is one of the first faculty members I approached for guidance when I just came to WVU. Despite his busy schedule, he gave appointment to meet him and provided valuable initial guidance to boost my confidence.

I am fortunate to have many friends and well-wishers at WVU who are always there for me throughout my graduate studies. Without their support, I would not come this far. Last but not the least, I remain indebted to my parents for their love and sacrifice. After all, nothing would have been possible for me without their support and encouragement. 


\section{List of Figures}

Figure 1.1 Example application of object tracking, video based face recognition........... 1

Figure 1.2 Application of object tracking in surveillance. Image adopted from [6] ...........2

Figure 1.3 Challenges in object tracking.......................................................... 3

Figure 1.4 Components of a typical object tracking algorithm................................. 4

Figure 1.5 Top ten probable windows containing an object in an image. ..................... 9

Figure 2.1 Dynamic model of IVT ........................................................................

Figure 2.2 Drift to Background of IVT: Representative Frames: Sylvester sequence. ....18

Figure 2.3 Drift to Background of IVT: Representative Frames: Coke Can sequence... 19

Figure 2.4 Drift to Background of IVT: Representative Frames: Bird 2 sequence ..........20

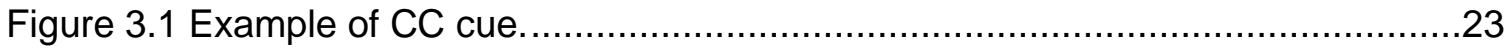

Figure 3.2 (a) Input frame from coke video sequence (b) Superpixel Segmentation $\quad .25$

Figure 3.3 (a) Input image (b) Superpixel segmentation (c) SS cue...........................26

Figure 3.4 Example illustrating Objectness Measure ...............................................27

Figure 3.5 Example of samples considered. Image adopted from [47].......................28

Figure 3.6 Cumulative distribution of weights. Image adopted from [47] .....................29

Figure 3.7 Example 1: Thresholding no of unique particles (nup) at resampling stage...30

Figure 3.8 Example 2: Thresholding no of unique particles (nup) at resampling stage...31

Figure 3.9 Implicit Tracking Failure Detection ......................................................33

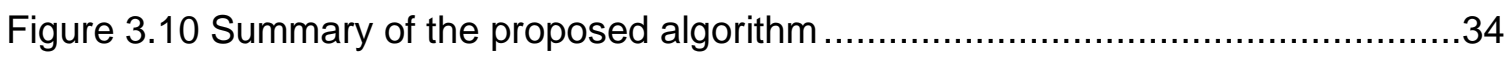

Figure 3.11 Block diagram of proposed tracking system .........................................

Figure 3.12 Proposed tracking system: Illustrative frames .......................................36

Figure 4.1 First frames of the tracking video sequences used ...............................41

Figure 4.2 Qualitative Assessment: Representative Frames: Coke Can sequence........42

Figure 4.3 Qualitative Assessment: Representative Frames: Basketball sequence. .....43

Figure 4.4 Qualitative Assessment: Representative Frames: Bird 2 sequence...........44

Figure 4.5 Qualitative Assessment: Representative Frames: Sylvester sequence........45

Figure 4.6 Computation of tracking success score...............................................46

Figure 4.7 Quantitative Assessment: Coke Can Sequence.......................................47

Figure 4.8 Quantitative Assessment: Bird 2 Sequence. ........................................47

Figure 4.9 Quantitative Assessment: Basketball Sequence. ...................................48 
Figure 4.10 Quantitative Assessment: Sylvester Sequence...................................48

Figure 4.11 Comparison of different online trackers: Coke Can Sequence. ..................52

Figure 4.12 Comparison of different online trackers: Bird 2 Sequence...........................52

Figure 4.13 Comparison of different online trackers: Basketball Sequence. ..................53

Figure 4.14 Comparison of different online trackers: Sylvester Sequence. ...................53

Figure 4.15 Some cases in which proposed approach failed .....................................55

\section{List of Tables}

Table 1.1 Components of Incremental Learning for Visual Tracking Algorithm. ............ 8

Table 4.1 Sequences used and challenging factors along with their sources.................39

Table 4.2 Quantitative Comparison: Success Rates of IVT and proposed method ........49

Table 4.3 Comparison of different online trackers: Success rates...............................54 


\section{Contents}

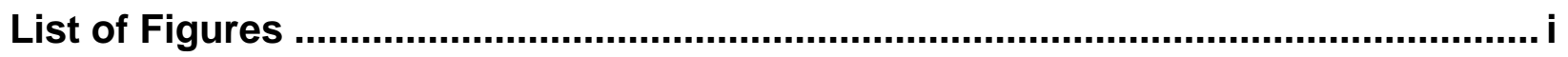

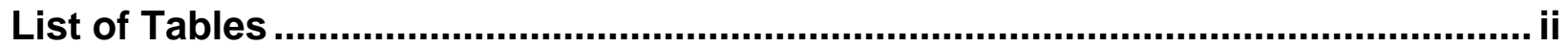

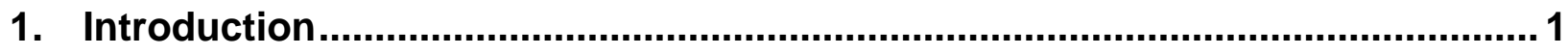

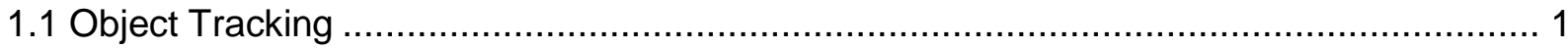

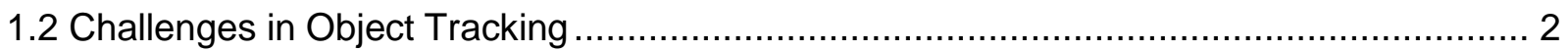

1.3 Components of Object Tracking Algorithm............................................................... 4

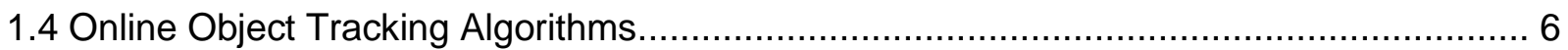

1.5 Incremental Learning for Visual Tracking Algorithm ................................................ 7

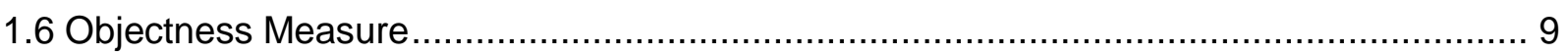

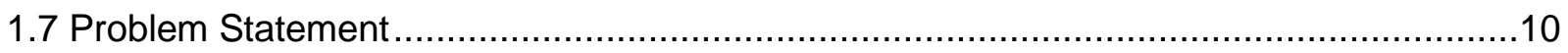

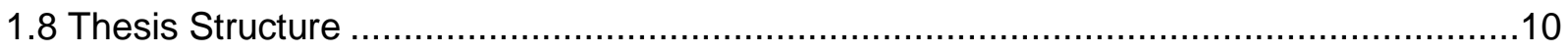

2. Incremental Learning for Visual Tracking ...................................................... 11

2.1 Appearance Model .........................................................................................

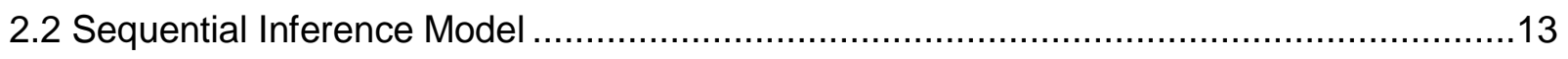

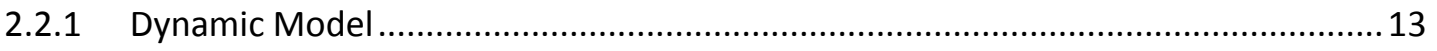

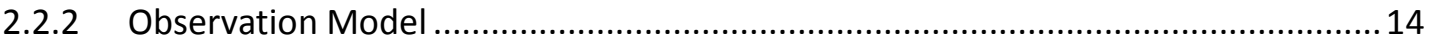

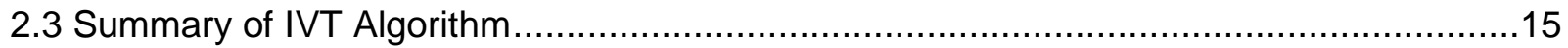

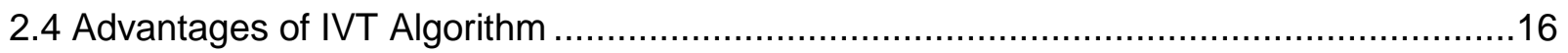

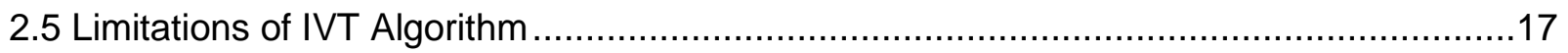

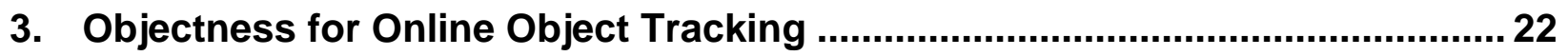

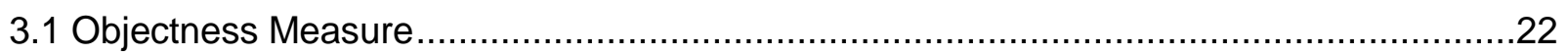

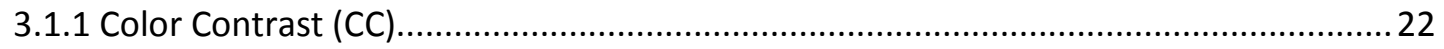

3.1.2 Superpixel Straddling (SS) ...................................................................................

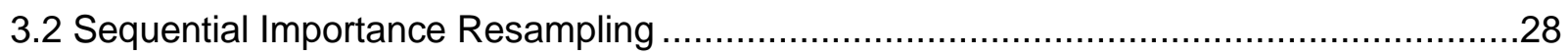

3.3 Objectness in the Context of Online Object Tracking:..............................................32

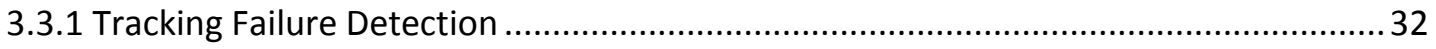

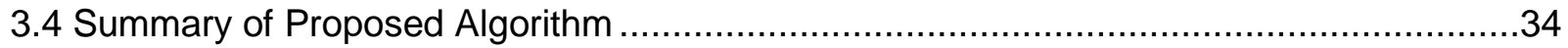

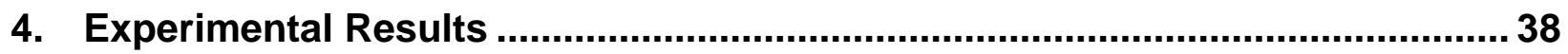

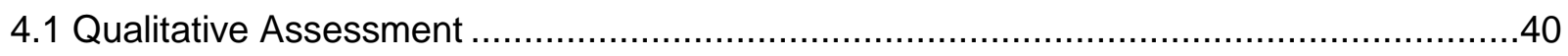




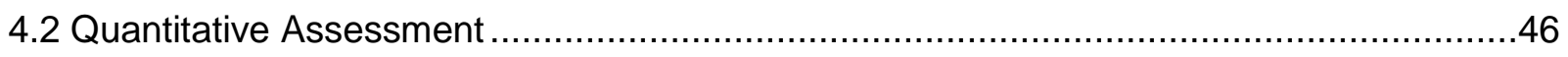

4.3 Comparison with Other Online Tracking Algorithms .............................................50

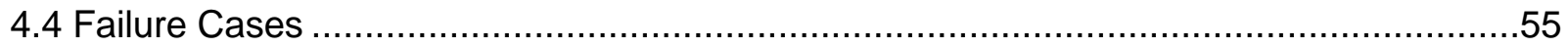

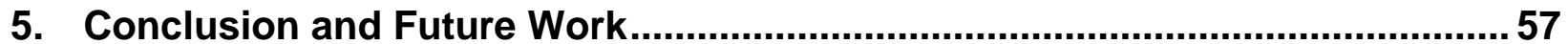




\section{Introduction}

\subsection{Object Tracking}

The objective of object tracking is to estimate the location of an object in continuous sequence of images given the position of the target in the first frame as input [1]. Object tracking is a very important problem in computer vision which has gained significant attention from the research community over the past decade. Object tracking is considered to be fundamental to many other vision problems such as recognition [2], surveillance [3], human computer interaction [4] and medical image analysis [5] to name a few.

Example application of object tracking, where face tracking module is employed as first stage of video based face recognition system [2] is shown in the Figure 1.1. Here performance of the face recognition module directly depends upon the success of face tracking, since the output from tracking module is fed as input to the recognition module. Another example application of tracking in a surveillance scene is shown in Figure 1.2.
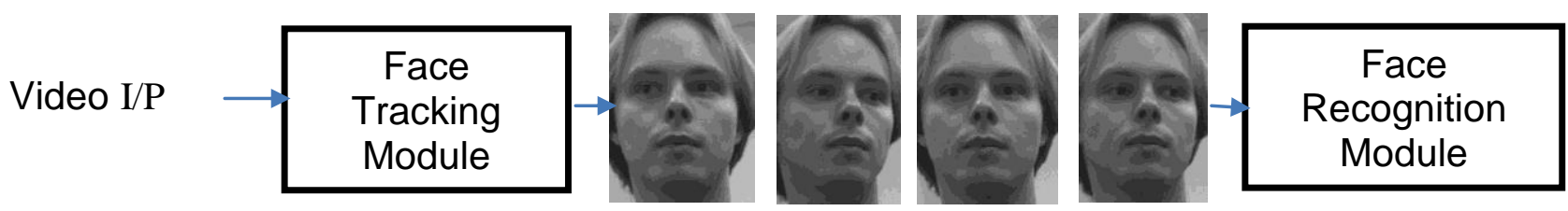

Figure 1.1 Example application of object tracking, video based face recognition. 


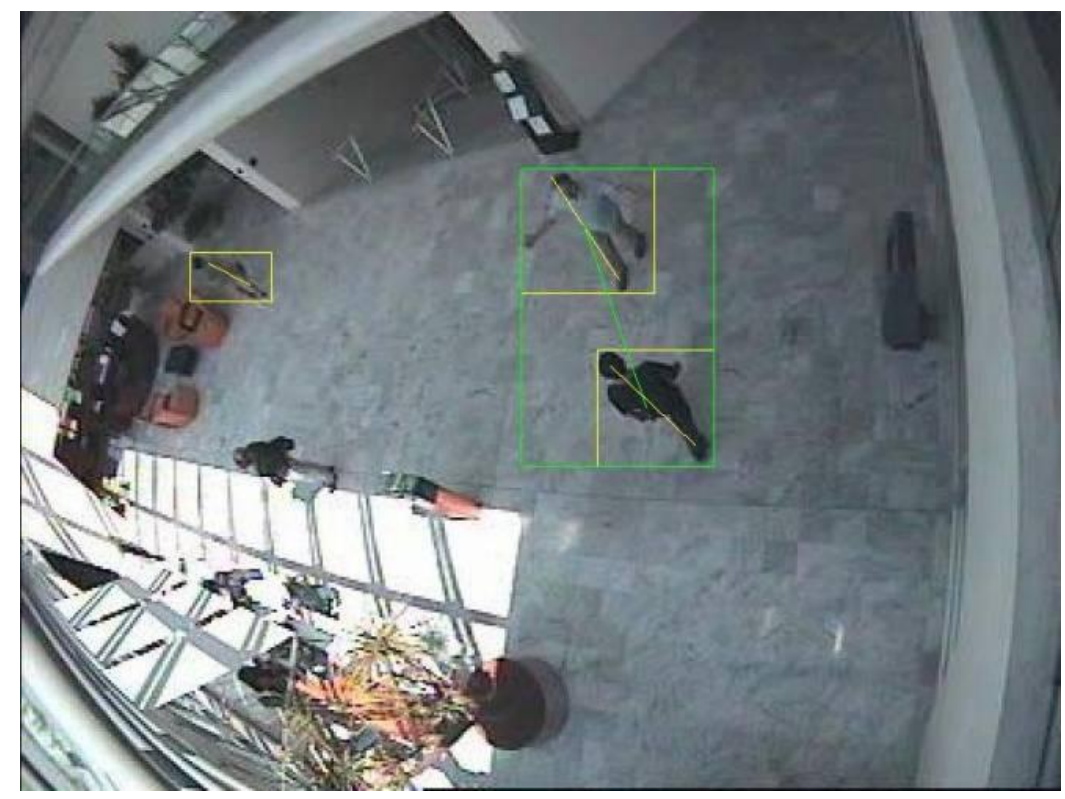

Figure 1.2 Application of object tracking in surveillance. Image adopted from [6]

\subsection{Challenges in Object Tracking}

Despite some success in building trackers of specific objects like faces, persons, cars etc., tracking generic objects is still very challenging due to the changes in the appearance (e.g. scale, deformation, rotation) of the objects and extrinsic factors like change in the illumination conditions of the scene, occlusion, presence of similar objects etc., [7]. Also, as general object tracking does not assume any domain knowledge, it further contributes to the difficulty in solving the problem. Some of the challenges while solving the object tracking problem like illumination conditions, occlusion and nonlinear deformation are depicted in the following Figure 1.3. 

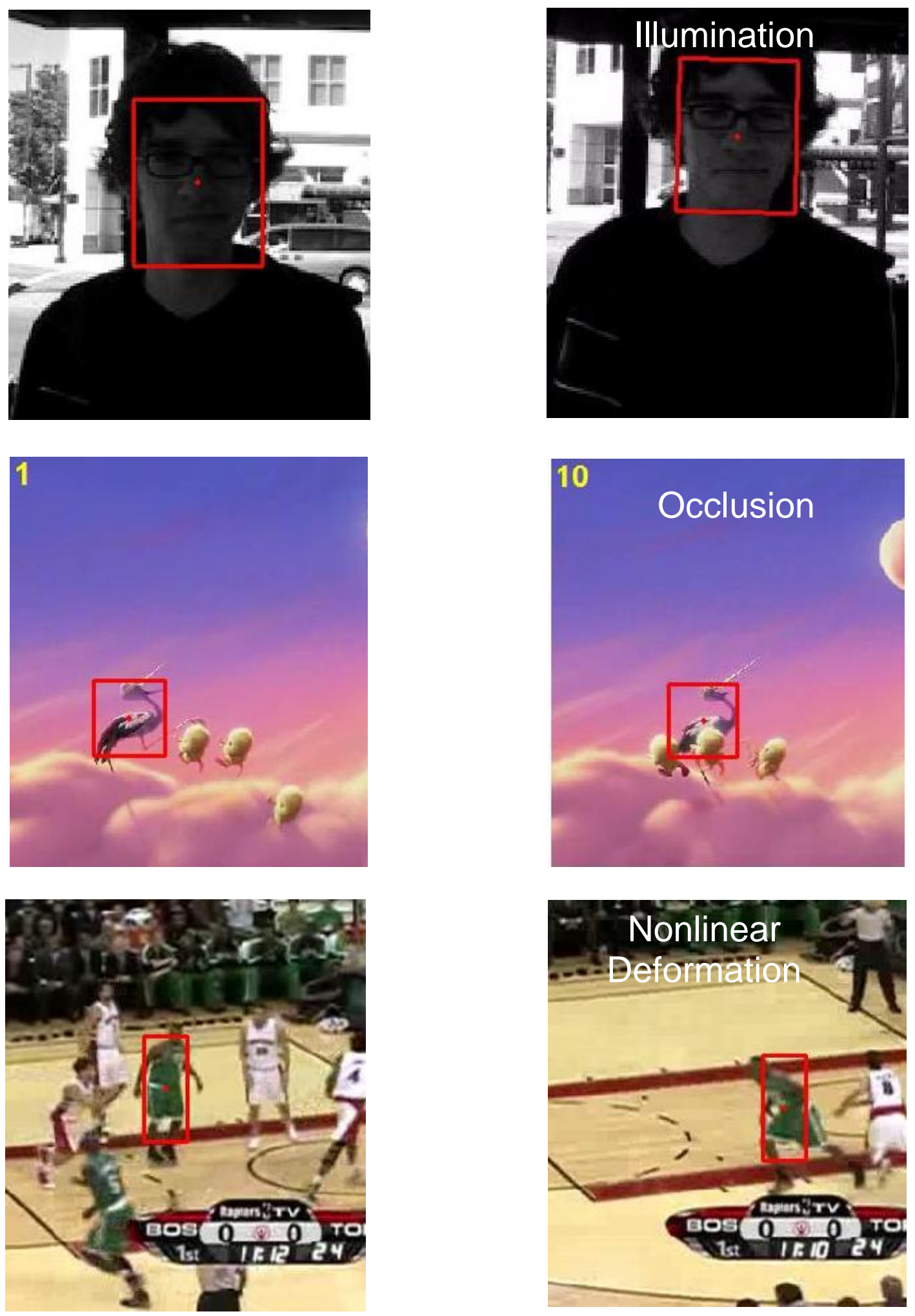

Figure 1.3 Challenges in object tracking. 
To cope up with the above mentioned challenges, adapting to the changes in the appearance of the target and scene conditions like illumination is one of the desired properties of any object tracking algorithm [2]. Several approaches to tracking have been reported in the literature. For instance, robust visual tracking of articulated objects using view based representation is considered in [8] and contour models are considered in [9], kernel based object tracking is employed in [10], robust online appearance mixture model is used in [11]. In addition to these methods, supervised discriminative methods for classification and regression as in [12]are exploited for visual tracking. This method demonstrated significant success in tracking specific objects like cars, but the computational complexity for training a support vector machine (SVM) is very high [13]. Each of these methods differ in their approach in solving object tracking in variety of ways. Typical components of any object tracking algorithm which can be modified to formulate different approaches for object tracking are discussed in the next section.

\subsection{Components of Object Tracking Algorithm}

Typically, any object tracking algorithm constitutes the following components [7]. (i) an appearance model, which computes the likelihood of object of interest being at particular position in the frame;(ii) Motion model which relates the location of object from frame to frame ; and (iii) Searching strategy in finding most likely position of the object in the present frame. These are depicted in the following Figure 1.4.

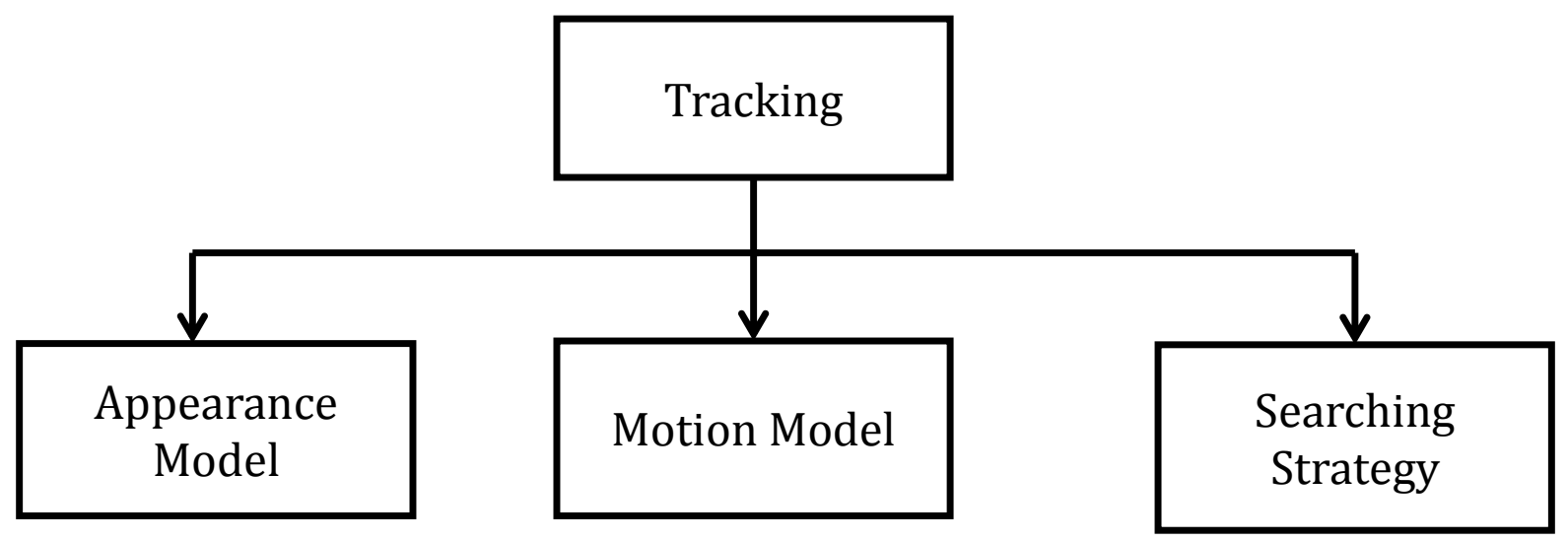

Figure 1.4 Components of a typical object tracking algorithm. 
The primary challenge of object tracking is to cope up with the constant variation of the appearance of the target object throughout the video. The appearance changes can occur due to both the intrinsic factors like variation in pose and shape deformation of the object or extrinsic factors such as change in illumination conditions in the scene, camera movement etc., [13].

Hence effective appearance model is one of the important components of any object tracking algorithm. The appearance of the target object can be modeled offline before task of the tracking begins i.e. models are trained only with appearance data of the object available before tracking. The tracking algorithms employing this type of appearance models may work well for short durations, but suffer from poor performance while tracking object for longer durations thus leading to the instability in the tracking performance. This is as a result of the adopted fixed appearance model which doesn't model the appearance and shape changes that are made available during tracking. Some of the examples of tracking algorithms that adopted offline appearance models in the literature include [8] where object representation based on view based appearance models was used, employing contours of the objects as in [9], color histogram based schemes as in [10],support vector machines as in [9].

Based on the type of motion model employed in the tracking algorithm dimensionality of the state vector $X_{t}$ can be derived. Motion models can be translational motion (2 parameters), similarity transform (4 parameters) or affine motion (6 parameters) [1]. For instance, well known kanade-lucas-tomasi [14] algorithm adopts a translational motion model to assess the object location from frame to frame. Tracking approaches proposed in [13] [15], adopts affine motion to relate the object location from frame to frame which can deal with scale changes and out of plane rotations of the target object.

Depending on the searching strategy used, object tracking algorithms can be classified as either stochastic or deterministic [1]. To obtain the tracking result in a frame, an objective function is minimized based on similarity, distance or classification measure [1]. In the case of the deterministic methods for searching the object, approaches based on gradient descent can be used to obtain the tracking result. Although this methods have an advantage of less computational complexity, they have limitation of getting stuck in the local minima [1]. Kalman filter [16] is a deterministic approach which assume a linear dynamic system for estimation. To deal with nonlinear and non-Gaussian models most of the latest tracking systems [13] [15] employs stochastic methods with particle filter to estimate the likely state of the objects. 
To overcome the limitations of the offline appearance learning models and to effectively adapt to the variations of the target object, several state of the art tracking algorithms employ online appearance models. In this type of models, appearance of the object is learnt on the fly thus making use of the changes in appearance and shape of the object that are made available during tracking. Adapting the appearance model is not trivial task since the correct adaptation always requires the precise location of the object to be known in a given frame [17] . Not ensuring this condition may lead to accumulation of the tracking errors that gradually make tracker drift from the original targets eventually resulting in tracking failure.

\subsection{Online Object Tracking Algorithms}

As aforementioned, to overcome problems of traditional offline appearance models, most state of the art tracking algorithms employ online appearance models. Object tracking algorithms can be broadly classified as generative and discriminative [1] based on their formulation. Generative methods estimate the location of the target object by finding the region most akin to the reference model [1]. To effectively deal with the challenges of the object tracking most recent tracking algorithms adopt robust offline or online appearance models. A subspace model is learnt offline based on different views of the object in [8], while incremental principal component analysis is used as appearance model in [13] to effectively adapt to the changes in views of the object and illumination conditions in the scene. Visual tracking using I1minimzation is proposed in [15].A tracking approach in which the observation model is decomposed in to multiple observation models to cope up with the drastic changes in the appearance of the object is proposed by kwon et.al in [18].Tracking problem is solved by applying recognition by parts approach in [19]. Even though this algorithm deals with partial occlusion of the object, as the adopted motion model is similarity transform, this approach might not work well when object undergo in -plane rotations [1]. Although generative methods for visual tracking perform well, one disadvantage with such approach is, the rich scene information is not taken in to account while updating the template which might be useful in separating the foreground from background [1]. On the other hand, discriminative methods formulate tracking task as a binary classification problem i.e., to distinguish object from background.

Recently, Grabner et.al [20] proposed online boosting tracking where a classifier is trained online incrementally to separate object from the background via a novel online Adaboost feature selection method. Since, online adaptation may lead to the drift in the tracking; a semi- 
supervised approach which alleviates this problem significantly is introduced in [21]. Supervised and semi-supervised classifiers are carefully combined to build a real time tracking system in beyond semi-supervised tracking algorithm [22]. Collins et.al proposed online selection of discriminative features [23]. They consider initial appearance of the object while updating the target template in the appearance model of the object to alleviate drift that occur due to adaptive appearance models. Use of Multiple Instance learning instead of supervised learning to build a robust tracker is demonstrated in [7].

A tracking framework in which tracking, learning and detection are combined to track an object in the videos of longer durations is proposed by kalal et.al in [24]. A novel P-N learning paradigm is proposed to achieve this. Exploiting context information to differentiate similar objects in the scene in a discriminative tracking framework is studied in [25].

Using orientation energies of the pixels of the object to solve visual tracking is introduced in [26].Each of these methods differ in a variety of ways in which they formulate the tracking approach and every algorithm has its own advantages and limitations. Choosing a particular algorithm primarily depends on the application for which it is going to be employed. Most of the online tracking algorithms, if not equipped with detecting drift from original targets completely drift towards background or non-targets thus losing the target in subsequent frames in an image sequence. This is the primary challenge faced by many of the tracking algorithms that use online appearance models.

\subsection{Incremental Learning for Visual Tracking}

\section{Algorithm}

Incremental Visual Tracking (IVT) algorithm [13] is a robust tracking algorithm which adaptively adjusts to the changes in the target appearance and scene conditions, by learning a low dimensional subspace representation. In contrast to other Eigen tracking algorithms [8], incremental visual tracking algorithm doesn't need a training module and learns the Eigen bases online incrementally while tracking the object. This tracker works in the particle filtering framework.

Incremental visual tracker extends Sequential Karhunen-Loeve (SKL) algorithm presenting a new incremental Principal Component Analysis (PCA) algorithm that has the ability 
to update the Eigen basis and the mean given the training data. This algorithm also uses a forgetting factor which can be used to down weight the effect of earlier observations on the PCA model.

Table 1.1 Components of Incremental Learning for Visual Tracking Algorithm.

\begin{tabular}{|l|l|}
\hline Appearance Model & Incremental Principle Component Analysis \\
\hline Motion Model & Affine Transform \\
\hline Searching Strategy & Particle filter \\
\hline
\end{tabular}

SKL algorithm has a problem as it makes no attempt to account for the changes in sample mean of the training data which changes over time with the arrival of new data. This problem is overcomed in the IVT algorithm by following an approach in which at each update of the Eigen basis, new training data is added with an additional vector chosen meticulously to correct for the time varying mean.

As mentioned before, to moderate the balance between old and new observations forgetting factor is used in the incremental Eigen basis update. The logical reason behind the introduction of forgetting factor is in many vision problems like tracking, it is important to focus more upon recently acquired observations rather than old observations as recent observations correlate more with the changes in the appearance of the target. Also as the time progress, the observation history becomes too large to an extent where learner might stop adapting to the changes in the observation. To handle this situation, IVT employs a forgetting factor. For each update, previous Eigen values are multiplied by a scalar factor $f \in[0,1]$ where $f=1$ indicates no forgetting to occur. This is one important contribution of this algorithm. More details of this algorithm are discussed in the chapter 2 of this thesis.

As many other algorithms that use adaptive appearance models, IVT suffers from drift while updating the template and as a result of challenges that were faced in the course of object tracking, it might lose the target and drift towards the background thus completely failing to track the object in subsequent frames. To alleviate this problem objectness measure has been introduced as an additional cue to appearance model of the tracker in this work. 


\subsection{Objectness Measure}

Objectness measure [27] estimates, the probability for any image window to contain object of any class. This measure combines many cues which denote the properties of any generic object in a Bayesian framework. The intuition behind this approach is, objects of any class are unique things with a closed boundary characteristic and center viz., cars, cows, airplanes, in contrast to the amorphous background material like sky, grass and road.

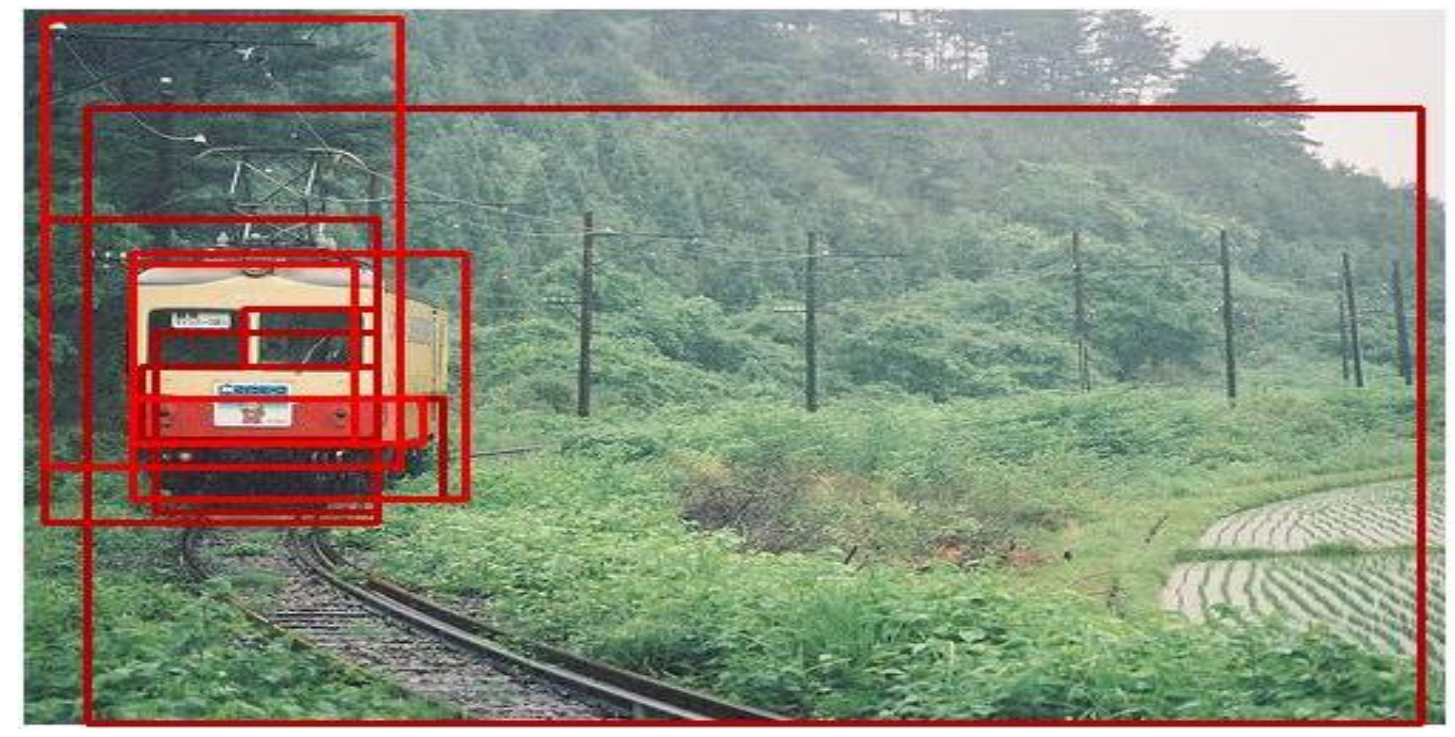

Figure 1.5 Top ten probable windows containing an object in an image returned by objectness measure.

For instance, in the image shown in Figure 1.5, train stands out from the surrounding based on certain characteristics that differentiate it from the background. Also, the top ten probable windows of containing an object according to objectness measure are depicted in the figure, where most of them lie on the train as it is the object in the scene. Objectness measure is used in an application to speed up the object detection in [27]. In this work, objectness measure is introduced as an additional cue in conjunction with the likelihood term of Incremental Visual Tracking (IVT) algorithm to alleviate drift to background problem of the tracker. 


\subsection{Problem Statement}

Many of the online tracking algorithms might fail to track an object due to sudden changes in the appearance of the object, gradual accumulation of tracking errors that results in drift from the original target (as a result of adaptive appearance model)or other challenges that occur in the course of tracking. Contribution of this thesis is to propose a tracking framework in which appearance model of IVT is aided with additional likelihood term (objectness measure), which quantifies how likely it is for a window to contain object of any class [28]. This helps the tracker from drifting to the background completely in the situations of sudden changes in illumination conditions in the scene, change in the pose, and scale of the target or other challenges like occlusion. Use of dynamic objectness for object tracking has been studied via discriminative approach in [29] (where both foreground and background were learnt). Here, we explore the effect of objectness in a generative approach (where only appearance of object is modeled) under the condensation framework.

\subsection{Thesis Structure}

Incremental Learning for Visual Tracking (IVT) algorithm is briefly discussed in chapter 2. Proposed combined framework of IVT and objectness measure is discussed in chapter 3. Both qualitative and quantitative experimental results were presented in chapter 4 of the thesis. This also includes some failure cases of the proposed approach. Finally in chapter 5 , conclusion and potential future work along this direction were discussed. 


\section{Incremental Learning for Visual Tracking}

Incremental learning for visual tracking algorithm is robust tracking algorithm in which an appearance model based on Incremental Principal Component Analysis is proposed in a particle filtering framework. The algorithm is proven to be capable of dealing with the sudden changes in the illumination conditions in the scene and pose changes of the object. It was designed to deal with the affine motion of the object since affine transform is adopted as motion model in the algorithm. Many of the details provided here are directly derived from [13].

\subsection{Appearance Model}

As aforementioned, Incremental PCA model is used in the IVT algorithm to learn and adapt to the changes in the appearance of the object incrementally. Typically, Eigen bases in PCA model is learnt offline by calculating the Eigen vectors of the covariance matrix $\frac{1}{n-1} \sum_{i=1}^{n}\left(I_{i}-\bar{I}\right)\left(I_{i}-\bar{I}\right)^{T}$, where $\left\{I_{1}, I_{2}, I_{3}, I_{4}, \ldots \ldots I_{n}\right\}$ denotes the training images and $\bar{I}=\frac{1}{n} \sum_{i=1}^{n} I_{i}$ denotes the sample mean of the training images. The equivalent operation is to perform singular value decomposition $U \sum V^{T}$ of the centered data matrix.

To adapt the appearance model to the new views of the target object, the PCA model has to be retrained with additional $m$ images for an arbitrary value $m$ ( $m$ depends on how frequently the appearance model is updated). Since performing this operation naively is computationally inefficient and not suitable for online learning in applications like object tracking, IVT algorithm employs efficient update mechanism by extending Sequential Karhunen-Loeve (SKL) algorithm proposed by Levy and Lindenbaum [30]. The specific advantage of this algorithm over naïve approach is to have space and time complexity constant in the number of training data that has been observed so far. Summary of the SKL algorithm is provided below.

Consider a $d x n$ matrix $A=\left\{I_{1}, I_{2}, I_{3}, I_{4}, \ldots \ldots I_{n}\right\}$. Here, $I_{i}$ denotes an observation for which singular value decomposition $A=U \sum V^{T}$ was already calculated. With the arrival of new 
observations denoted by a $d x m$ matrix $\mathrm{B}$, SVD of the concatenation of $\mathrm{A}$ and $\mathrm{B}$ is computed as $[A B]=U^{\prime} \Sigma^{\prime} V^{\prime T}$. Considering $\tilde{B}$ an orthogonal component of $\mathrm{B}$ to $\mathrm{U}$, the concatenation can be expressed in partition form as given below

$$
[A B]=\left[U \begin{array}{c}
\tilde{B}
\end{array}\right]\left[\begin{array}{cc}
\sum & U^{T} B \\
0 & \tilde{B}^{T} B
\end{array}\right]\left[\begin{array}{cc}
V^{T} & 0 \\
0 & I
\end{array}\right]
$$

Let, $R=\left[\begin{array}{cc}\sum & U^{T} B \\ 0 & \tilde{B}^{T} B\end{array}\right]$,which is a square matrix of dimensions $k+m$ where $\mathrm{k}$ denotes no of singular values in $\sum$ and $m$ denotes the no of new observations at an update. SVD of R, given by $R=\widetilde{U} \widetilde{\Sigma} \tilde{V}^{T}$ can be calculated in same time irrespective of the value of $\mathrm{n}$ which denotes the initial number of observations. Now, SVD of $[A B]$ can be established by the following relation

$$
[A B]=\left(\left[\begin{array}{lll}
U & \widetilde{B} & \widetilde{U}
\end{array}\right) \sum \widetilde{V}^{T}\left[\begin{array}{cc}
V^{T} & 0 \\
0 & I
\end{array}\right]\right)
$$

Here the values that needs to be computed are $\widetilde{U}^{\prime}$ and $\Sigma^{\prime}$ from the SVD of $[A B]$. Hence SKL algorithm can be formulated in the following steps.

1. $\Sigma$ and $U$ are given from SVD of $A$. With the arrival of new data(denoted by matrix $B$ ) $U^{\prime}$ and $\Sigma^{\prime}$ are computed from SVD of concatenation of A \& B i.e. [A B].

2. $\tilde{B}$ and $R$ are obtained by computing $Q R$ decomposition of $\left[U \sum B\right]$.

3. SVD of $R$ is computed.

4. In the final step $U^{\prime}=\left[\begin{array}{ll}U & \widetilde{B}\end{array} \widetilde{U}\right.$ and $\Sigma^{\prime}=\widetilde{\Sigma}$. Only desired no of basis vectors and singular values can be retained at this step discarding excessive vectors and singular values.

There are many other sophisticated algorithms in literature that are developed to efficiently update the Eigen bases with the new data that arrives incrementally [31] [32] [30] [33]. However, most of these algorithms does not update the mean or presume the data is inherently zero mean neither of which is appropriate for tracking application. IVT algorithm takes care of this problem by extending SKL algorithm outlined above with an efficient update of the Eigen bases along with the change in the mean with the arrival of new data. As mentioned in chapter 1 , the algorithm also employs a forgetting factor which helps to focus more upon the recent observations rather than older observations to maintain greater adaptivity to the changes in the appearance of the object. A forgetting factor denoted by $f$ is incorporated in each incremental basis update. For each update previous Eigen values are multiplied by scalar factor $f \in[0,1]$, 
where $f=1$ indicates no forgetting to occur. Authors of IVT algorithm has proved the efficacy of using forgetting factor in the incremental update both theoretically and empirically.

\subsection{Sequential Inference Model}

Tracking problem in IVT algorithm is considered to be an inference task in Markov model with hidden state variables. Here state variable is denoted by $X_{t}$ which describes the affine motion parameters of the target at time $t$ i.e. location of the target at time $t$. Given a set of observed images $I_{t}=\left\{I_{1}, I_{2}, \ldots \ldots, I_{t}\right\}$ the aim is to estimate the value of the hidden state variable $X_{t}$. Using Bayes theorem the following relation can be established

$$
p\left(X_{t} \mid \underline{I_{t}}\right) \alpha p\left(I_{t} \mid X_{t}\right) \int p\left(X_{t} \mid X_{t-1}\right) p\left(X_{t-1} \mid I_{t-1}\right) d X_{t-1}
$$

Here tracking process can be attributed as a combination of observation model $p\left(I_{t} \mid X_{t}\right)$ where likelihood of $X_{t}$ is estimated observing $I_{t}$, and the dynamic model between the two states $p\left(X_{t} \mid X_{t-1}\right)$. A form of condensation algorithm employed in [9] is used to model the distribution over the object's spatial location, as it evolves over time.

\subsubsection{Dynamic Model}

The position of the target object in an image frame is indicated by the affine image warp. This warp transforms the coordinate axis in such a way that the target object lies in a unit square. IVT uses affine transformation for dynamic model with 6 parameters. Let $X_{t}$ indicates the location of the object at time $t$, then $X_{t}=\left(x_{t}, y_{t}, \theta_{t}, s_{t}, \alpha_{t}, \emptyset_{t}\right)$ where $x_{t}, y_{t}, \theta_{t}, s_{t}, \alpha_{t}, \emptyset_{t}$ denotes $x, y$ translation, rotation angle, scale, aspect ratio and skew direction at a time instant $t$ respectively.

The motion between the frames is an affine transformation with each parameter in $X_{t}$ modelled independently using Gaussian distribution over its past position in $X_{t-1}$. The relation can be given as

$$
p\left(X_{t} \mid X_{t-1}\right)=N\left(X_{t} ; X_{t-1}, \varphi\right)
$$

Here $\varphi$ denotes the diagonal covariance matrix with the corresponding variances of the affine parameters as its elements viz. $\sigma_{x}^{2}, \sigma_{y}^{2}, \sigma_{\theta}^{2}, \sigma_{s}^{2}, \sigma_{\alpha}^{2}, \sigma_{\emptyset}^{2}$. These parameters are determined 
based on the expectation of how much a target might move from one frame to other frame. These parameters are fixed and will remain the same with the change in time. With larger values in the diagonal covariance matrix, and using more no of particles, tracking performance can be enhanced however the computational complexity of the algorithm would be increased. It is important to have a tradeoff between the effectiveness and efficiency which can be achieved by careful tuning of the algorithm parameters.

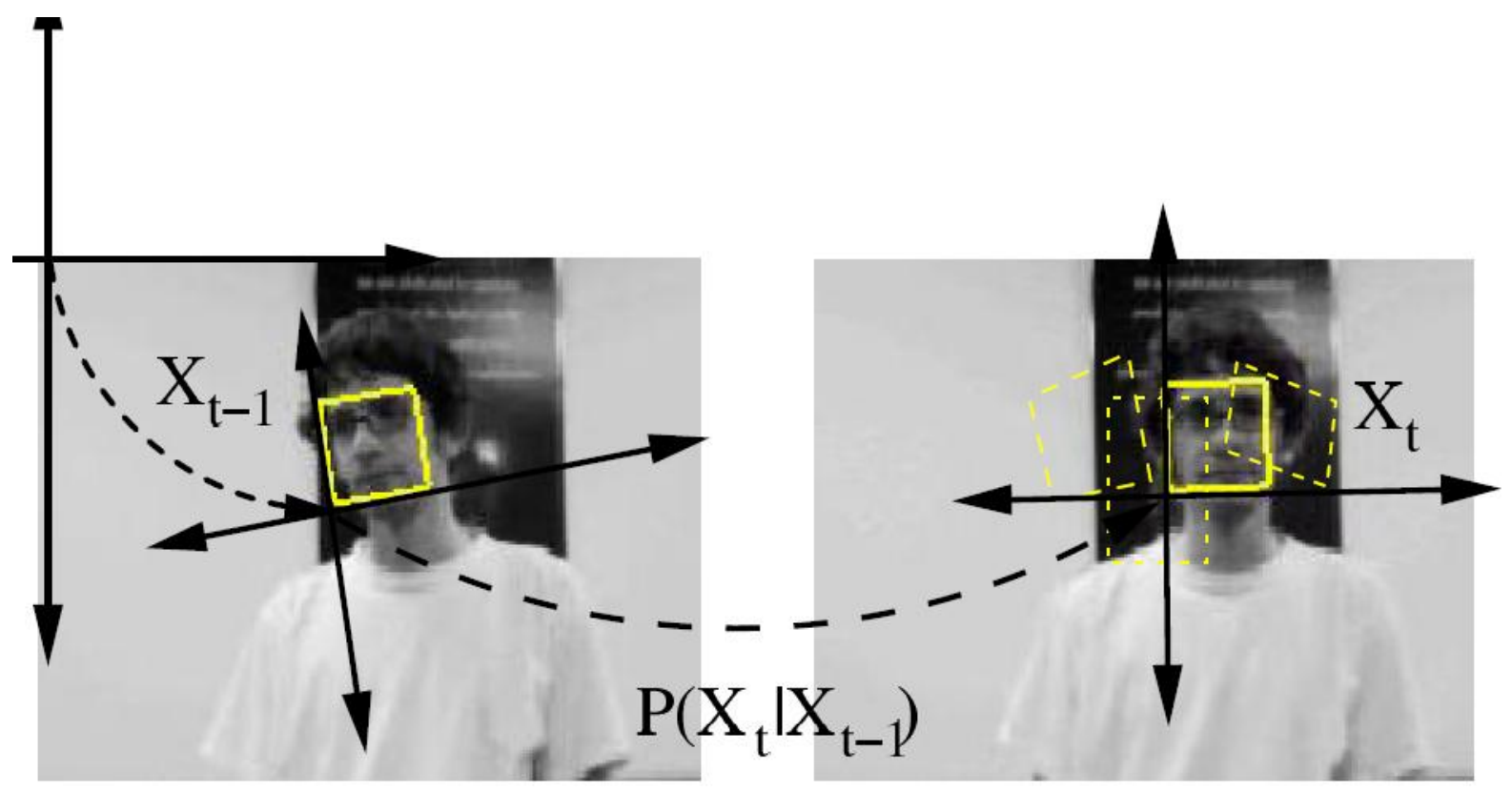

Figure 2.1 Dynamic model: Target location is represented by an affine transformation that warps the coordinate system so that target lie with in the unit square. Particles are drawn according to dynamic model described above.Image adopted from [13]

\subsubsection{Observation Model}

In IVT algorithm, image observations are modelled using probabilistic principal component analysis [34]. Let an image patch be represented by $I_{t}$ estimated by $X_{t}$, it is assumed that image patch was generated from the subspace of the target object measured by $U$ and centered at mean $\mu$. Then the probability of an image patch to be generated from this subspace is inversely proportional to the distance $d$ of the patch to the reference point of the subspace. This distance can be further decomposed in to distance to the subspace denoted by $d_{t}$ and the distance within the subspace $d_{w}$.

Let the probability of an image patch generated from a subspace, denoted by $p_{d_{t}}\left(I_{t} \mid X_{t}\right)$,is modelled by a Gaussian distribution 


$$
p_{d_{t}}\left(I_{t} \mid X_{t}\right)=\mathrm{N}\left(I_{t} ; \mu, U U^{T}+\epsilon I\right)
$$

Here $I$ denotes an identity matrix, $\mu$ represents the mean and the term $\epsilon I$ denotes the additive Gaussian noise.

The probability of image patch within the subspace can be computed through mahalanobis distance (which takes covariance in to consideration). It is given by

$$
p_{d_{w}}\left(I_{t} \mid X_{t}\right)=\mathrm{N}\left(I_{t} ; \mu, U \Sigma^{-2} U^{T}\right)
$$

In this relation $\mu$ denotes the mean and $\Sigma$ is the matrix of singular values in correspondence to the columns of $U$.

Combining both the relations, the likelihood of an image patch to be generated from the subspace (that justifies the initial assumption) is given by

$$
p\left(I_{t} \mid X_{t}\right)=p_{d_{t}}\left(I_{t} \mid X_{t}\right) p_{d_{w}}\left(I_{t} \mid X_{t}\right)=\mathrm{N}\left(I_{t} ; \mu, U U^{T}+\epsilon I\right) \mathrm{N}\left(I_{t} ; \mu, U \Sigma^{-2} U^{T}\right)
$$

Hence given the sampled particle location $X_{t}$ and corresponding image patch $I_{t}$,observation likelihood is computed using the above equation.

\subsection{Summary of IVT Algorithm}

In this section we provide the summary of the IVT algorithm. As discussed before, IVT do not use any database offline to learn the appearance of the target object. Initial location of the target in the first frame is located manually. Incremental PCA is used to learn the changes online in the appearance of the object. Algorithm allows to tune different parameters such as update frequency of the appearance model and the number of particles used in estimating the posterior distribution. With more frequent updates, it is possible to model the drastic changes in the appearance of the object however the tracker then would me more prone to the drifts. With more number of particles there would be fine granularity in estimated samples thus leading to higher performance although the computational complexity would be increased. Hence it is important to have a tradeoff and choose the parameters accordingly.

Summary of the IVT algorithm is outlined in the table below. 
Table 2.1 Summary of IVT algorithm

Initialization: The initial location of the target in the first frame is located manually. Other parameters of the algorithm such as number of particles, and variances of the dynamic model, update frequency etc., can be chosen at the start of the algorithm.

\section{Tracking:}

1. Initialize the mean of the Subspace to be equal to the target patch extracted in the first frame based on manual input. Eigen basis is initialized to be empty initially.

2. Proceed to the next frame. Extract the samples from the particle filter according to the dynamic model as discussed in section 2.2.1.

3. Extract the image patches corresponding to the samples generated from particle filter and compute their likelihood weight according to observation model as discussed in section 2.2.2.

4. Store the image patch corresponding to the most likely sample (Maximum likelihood weight) and after desired number of observations are accumulated (based on the update frequency) update the appearance model (Incremental PCA algorithm) with the new observations, Eigen bases and mean.

5. Go to step 2.

\subsection{Advantages of IVT Algorithm}

Since IVT algorithm considers covariance of the pixels in the object region while modeling the appearance of the target object, it has notion of tracking a thing which can also facilitate object recognition [13]. Also as IVT uses incremental PCA model, it is effective in dealing with changes in illumination conditions of the scene and pose of the object. Moreover, IVT employs a Gaussian random walk model as a dynamic model with particle filter which makes it somewhat resilient to full occlusion (for brief periods) since tracker can search around for the target object after recovering from the occlusion [1]. 


\subsection{Limitations of IVT Algorithm}

As IVT algorithm employs holistic appearance model, it cannot deal with partial occlusion very well. Also, since it uses all the previous tracking results for the template update, gradual accumulation of tracking errors makes it susceptible to the drift from original targets to non-targets or background [1]. IVT does not have a mechanism to detect and correct the drift and once the tracker drifts completely to the background, there is no means to get the target back. Some of the cases in which IVT algorithm drifts completely to background as a result of the challenges faced during course of tracking are depicted in the Figures 2.1 to 2.3. Representative frames showing tracking performance on three sequences sylvester sequence obtained from Ross et.al [13],coke sequence obtained from Babenko [35] et.al and bird2 sequence obtained from Wang et.al [36] are shown in the examples. In the figures shown, red rectangle denotes the tracker and second row shows (considering left to right) current subspace center, tracked patch, reconstruction error and reconstructed image consecutively. Last row shows the top ten Eigen basis images of the current subspace. 

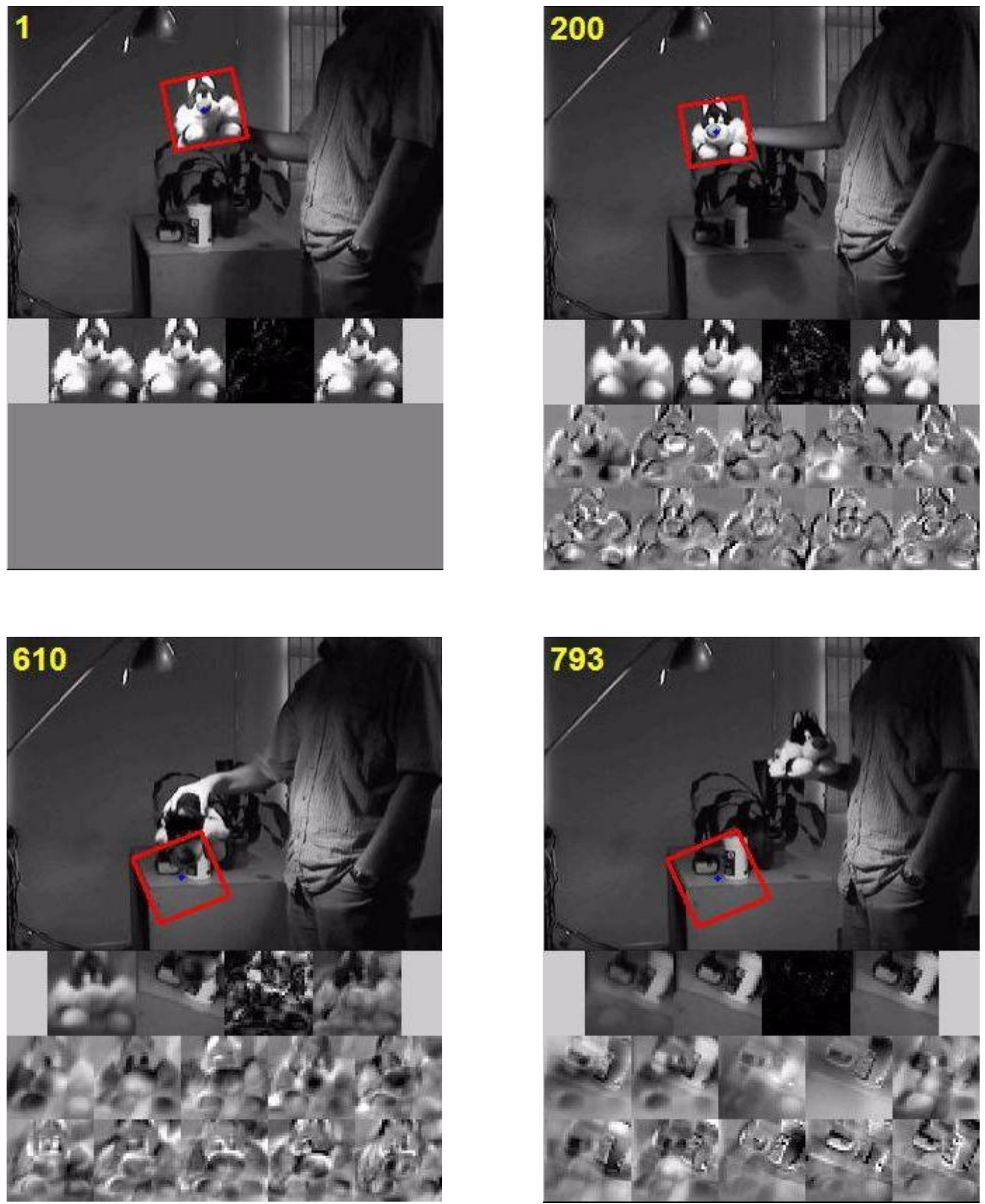

Figure 2.2 Drift to Background of IVT: Representative Frames: Sylvester sequence. Red rectangle denotes the tracker and second row shows (considering left to right) current subspace center, tracked patch, reconstruction error and reconstructed image consecutively. Last row shows the top ten Eigen basis images of the current subspace 

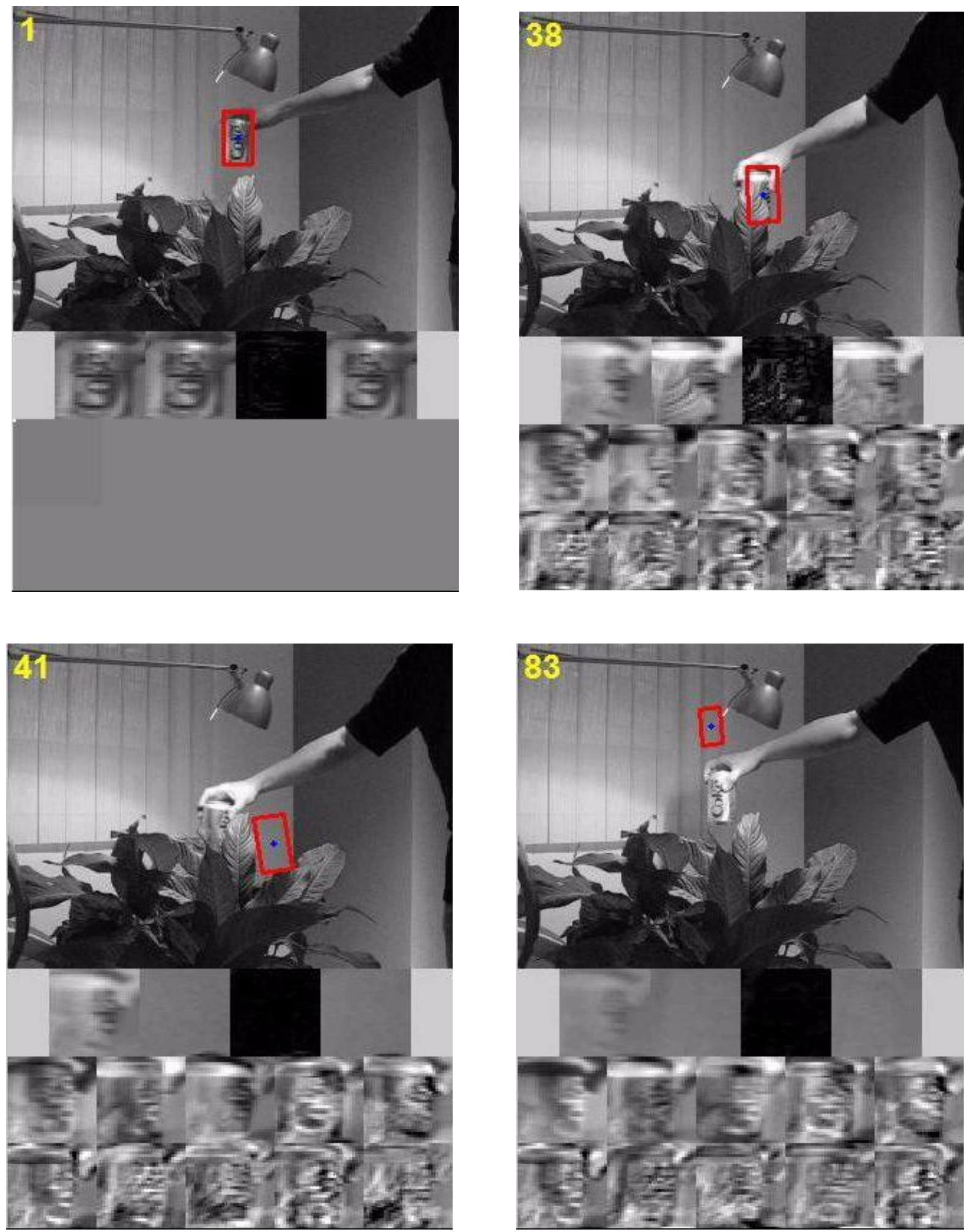

Figure 2.3 Drift to Background of IVT: Representative Frames: Coke Can sequence. Red rectangle denotes the tracker and second row shows (considering left to right) current subspace center, tracked patch, reconstruction error and reconstructed image consecutively. Last row shows the top ten Eigen basis images of the current subspace 

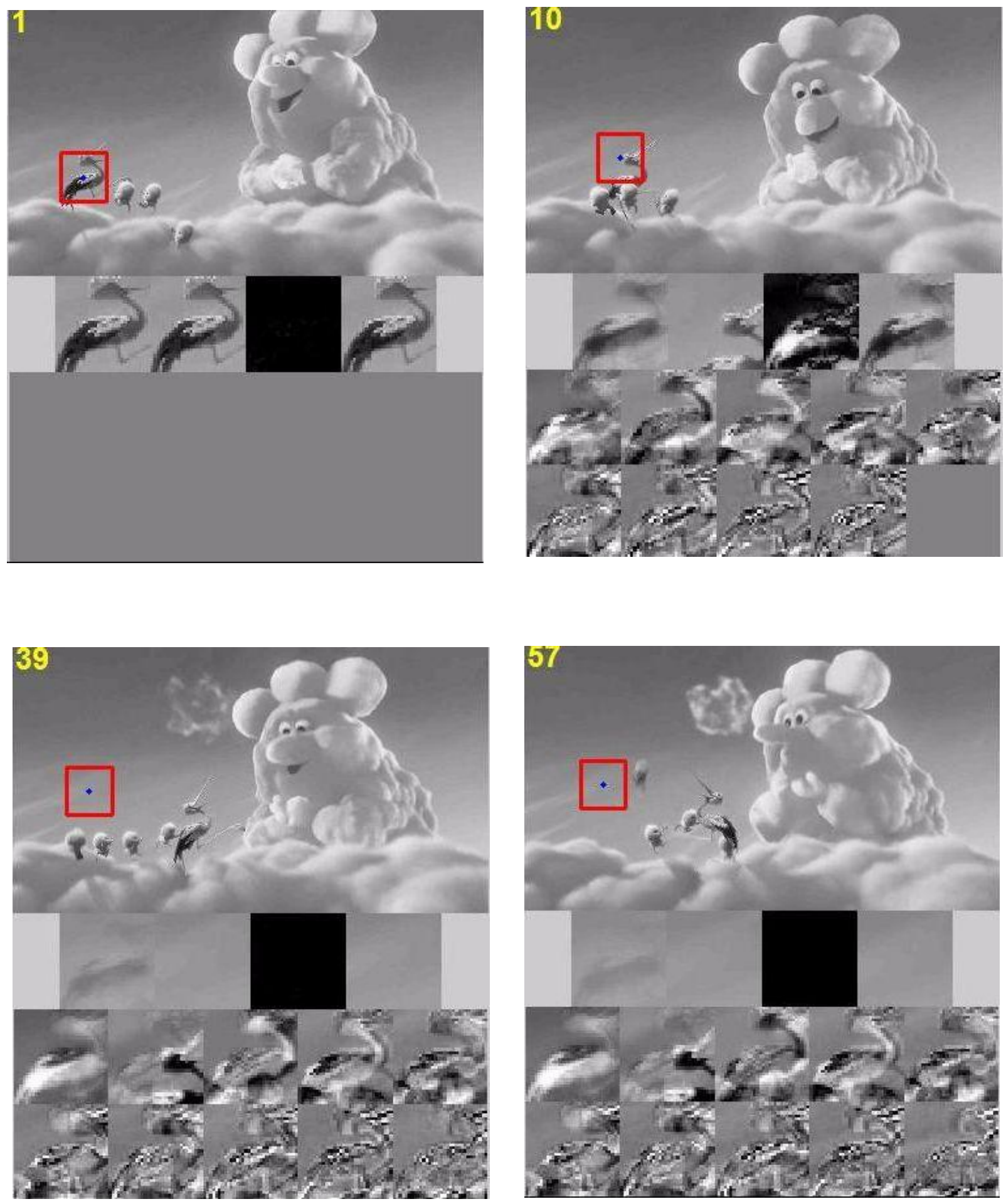

Figure 2.4 Drift to Background of IVT: Representative Frames: Bird 2 sequence. Red rectangle denotes the tracker and second row shows (considering left to right) current subspace center, tracked patch, reconstruction error and reconstructed image consecutively. Last row shows the top ten Eigen basis images of the current subspace 
As shown in the Figures above, IVT might drift to the background as a result of challenges faced during the tracking. In the first example (Figure 2.1) on Sylvester sequence [13], IVT tracker failed in the frame 610 may be due to extreme changes in illumination conditions of the scene and pose variations of the object [13]. In the later examples i.e., on coke [7] [35] and bird2 [36] sequences, tracker has failed as a result of the occlusion of the target. It is important to note that, IVT do not have a mechanism to detect such failures and tracker once drifted to background will lose the target completely in subsequent frames in the video sequence. In the next chapter, we discuss the proposed approach of incorporating objectness in to IVT algorithm to partially alleviate this problem. 


\section{Objectness for Online Object Tracking}

\subsection{Objectness Measure}

Objectness measure [37] [27] computes the probability of an image window to contain object of any class. This measure was developed by combining several different cues which are based on different attributes or characteristics of an object such as

(a) Uniqueness of the objects which makes them stand out from the surroundings (saliency) [38] [39] [40] [41].

(b) Objects may appear different from the background in the scene [42] [43].

(c) Most objects possess well-defined closed boundary characteristic.

In [27], different cues are combined in a Bayesian framework to obtain a final integrated score for a window to contain an object. As stated earlier, we have employed objectness measure to aid likelihood term of Incremental Learning for Visual Tracker's (IVT) appearance model, which might help in avoiding its drift towards the background thus improving algorithm's tracking performance.

We have used two different cues of an object proposed in [27] viz., Color Contrast (CC) which denotes different appearance characteristic of the object and Superpixel Straddling which measures the closed boundary characteristic of an object. These cues were combined in a Bayesian framework to obtain final integrated score of a window which denotes its objectness. These cues were discussed in the following sections.

\subsubsection{Color Contrast (CC)}

Color Contrast (CC) measures the local difference between patches of an image window and its surrounding area. Surrounding area of any window w, considered in the measure denoted by $\operatorname{Surr}\left(w, \theta_{C C}\right)$ is obtained by elongating the image window $w$ by $\theta_{C C}$ in every direction. The relation is established using the following equation $\frac{\left|\operatorname{Surr}\left(\mathrm{w}, \theta_{C C}\right)\right|}{|w|}=\theta_{C C}^{2}-1$. Then 
color contrast (CC) is measured by computing the histograms $h$ of both the windows in $L A B$ space. Chi-square distance between these histograms is used as similarity metric.

$$
C C\left(w, \theta_{C C}\right)=x^{2}\left(h(w), h\left(\operatorname{Surr}\left(w, \theta_{C C}\right)\right)\right.
$$

The intuition behind this cue is that an object generally appears differently from the background and thus possess a different color distribution when compared to the background, thus making $\mathrm{CC}$ to be higher for the windows containing the object.

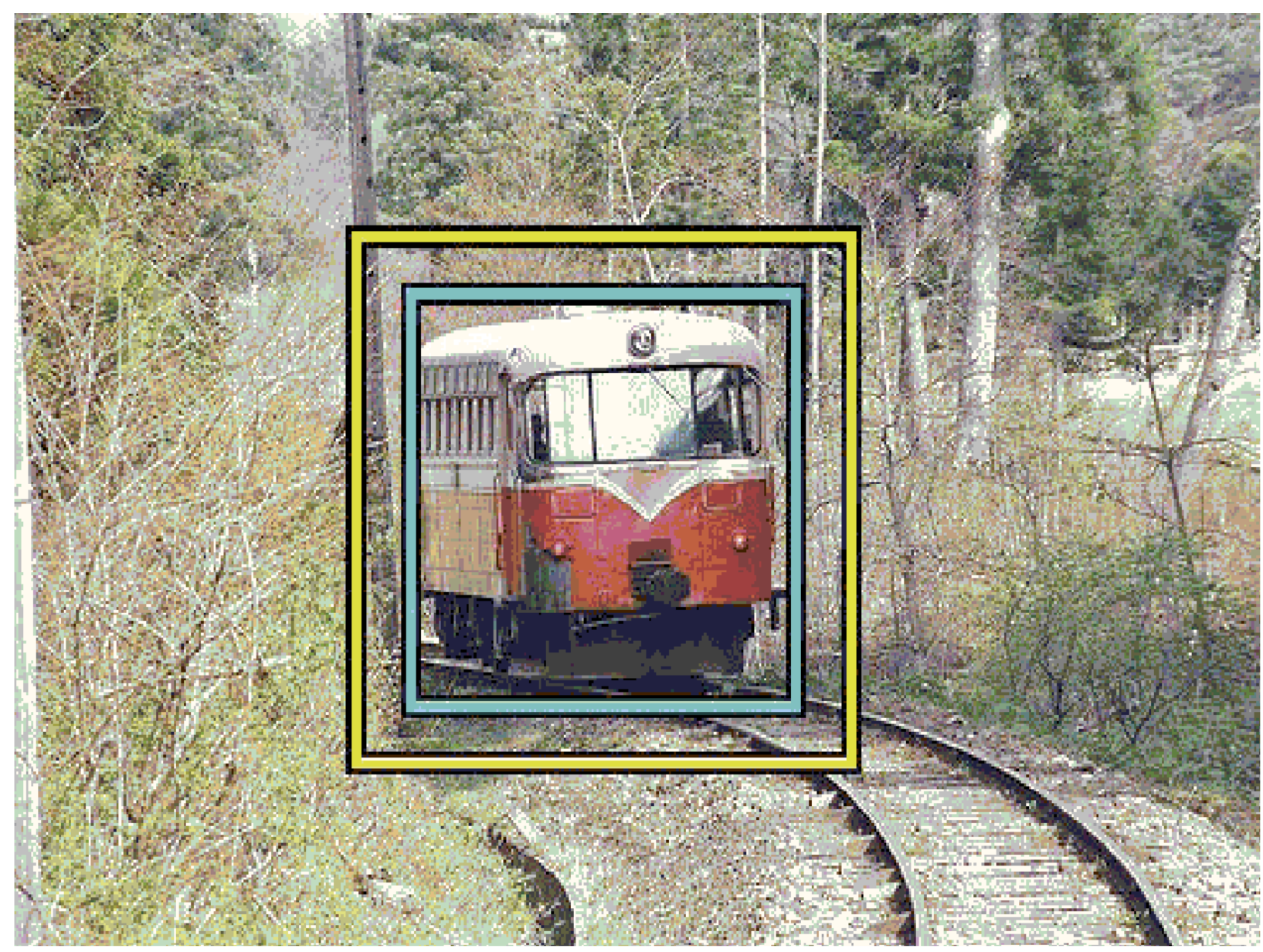

Figure 3.1 Example of CC cue. Window containing object (cyan) has higher color contrast than the surrounding area (denoted by yellow). Image adopted from [27]

\subsubsection{Superpixel Straddling (SS)}

Superpixel straddling (SS) [27] measures the closed boundary characteristic of an object. In this cue, image is segmented in to uniform regions of color or texture based on midlevel features superpixels [44]. An important property of the superpixels is to sustain object 
boundaries i.e., all the pixels in a superpixel typically belong to the same object [45]. This property is primarily used in Superpixel straddling (SS) cue to compute the probability of a window to contain an object.

A superpixel denoted by $\mathbf{S}$, is said to be straddling a window denoted by $w$, if it consists at least one pixel inside and one pixel outside the window. For instance, in the Figure 3.3, all the surface of the image window containing the object $(w 1)$ are overlapped by the superpixels containing inside it. On the other hand, surface of a window possibly lying partially on the background such as $w 2$ is overlapped by superpixels extending outside its region which are said to be straddling superpixels. Then SS cue is computed by the following relation

$$
S S\left(w, \theta_{S S}\right)=1-\sum_{s \in S\left(\theta_{S S}\right)} \frac{\min (|s \backslash w|,|s \cap w|)}{|w|}
$$

In the above equation $S\left(\theta_{S S}\right)$ denotes the superpixels with a segmentation scale $\theta_{S S}$ obtained through graph based segmentation approach proposed in [44] .Example of $S\left(\theta_{S S}\right)$ obtained using this procedure for first frame of coke sequence is shown in the Figure 3.2. Image shown in this figure is extracted from the coke sequence obtained from [7]. 


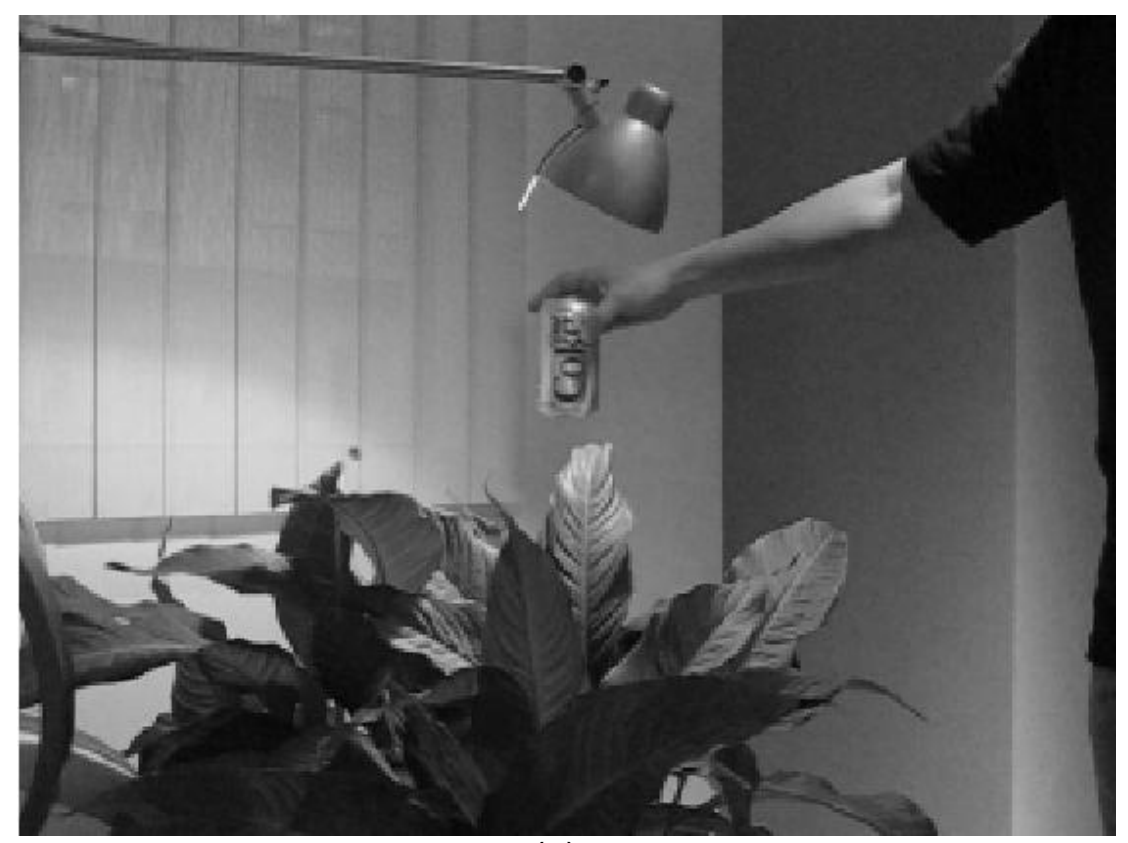

(a)

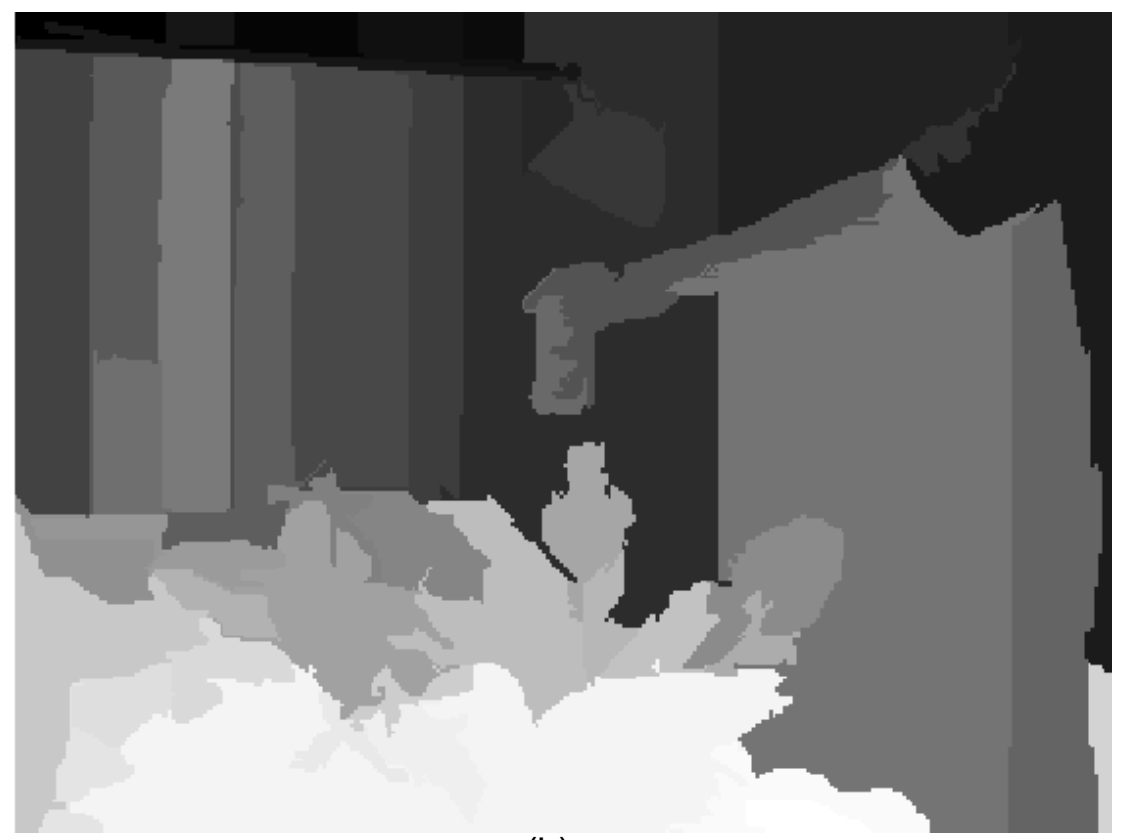

(b)

Figure 3.2 (a) Input frame from coke video sequence (b) Superpixel Segmentation obtained through [44]

For each superpixel $s$, where $s \in S\left(\theta_{S S}\right),|s \cap w|$ denotes the intersection of the superpixel with the window while $|s \backslash w|$ denotes the area of the window w outside the superpixel. 
Here superpixels lying completely inside or outside the window do not contribute to the sum in the equation (1) while for a superpixel which is straddling, the contribution is low when it is part of the object. On the contrary, the contribution of straddling superpixel $s$ to the sum is higher when it extends outside the window w. This approach assigns higher score to the windows fitting tightly around the object.

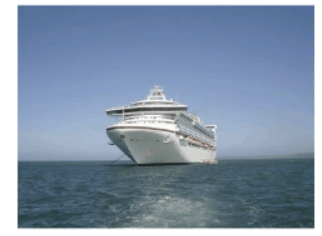

(a)

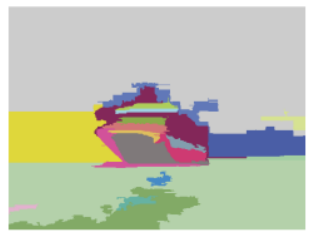

(b)

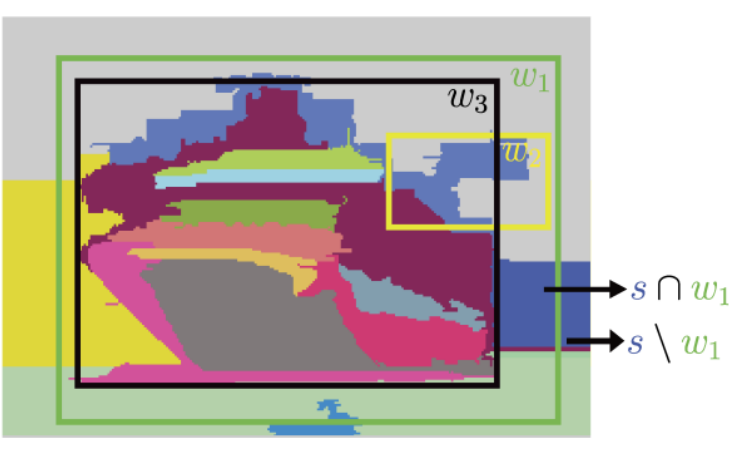

(c)

Figure 3.3 (a) Input image (b) Superpixel segmentation (c) SS cue. Here w3 will be scored highest (window tightly fitting the object) while w2 (window mostly lying on the background) will be scored lowest according to SS cue. Image adopted from [27]

The Parameters $\theta_{C C}$ and $\theta_{S S}$ in [27] are learnt in a Bayesian framework. A training dataset $\mathrm{T}$ containing 50 Images from several well-known datasets is formed. Initially, for each image in T, 100000 random windows are sampled by means of uniform distribution. Windows that cover the annotated object are taken as positive examples ( $W^{o b j}$ ), and rest of them are considered negative $\left(W^{b j}\right)$. Optimal $\theta_{C C}$ and $\theta_{S S}$ are determined by maximizing the posterior probability computed through building the likelihoods for positive and negative classes [27]. Here $\theta_{C C}$ is parameter for $\mathrm{CC}$ cue which define outer ring considered for given window $w$, $\operatorname{Surr}\left(w, \theta_{C C}\right)$ while $\theta_{S S}$ defines segmentation scale of the superpixels in [44].

Since color contrast and superpixel straddling are complementary cues each denoting different characteristics of an object, integrating them would be more reliable in computing the objectness of an image window [27]. Hence, in [27] these cues are combined in a naive Bayes model, assuming each cue is independent to obtain final integrated score for a window to contain an object. Training needs to estimate the priors $p(o b j), p(b g)$ which are computed based on relative frequency of background and object. Individual cue likelihoods $p(c u e \mid c)$ for 
cue $\in\{C C, S S\}$ and $c \in\{o b j, b g\}$ are learnt from huge set of training windows denoted by $W^{o b j}$ for objects and $W^{b g}$ for background.

In this work, for each frame of the video, possible windows containing target object are obtained from the particle filter of IVT [13], and the cues CC and SS [27] are computed for each window. Considering $A \in\{C C, S S\}$, these cues are combined to obtain posterior probability for a window using the well-known Bayes theorem as given below.

$$
\begin{gathered}
p(o b j \mid A)=\frac{p(A \mid o b j) P(o b j)}{p(A)} \\
=\frac{p(o b j) \prod_{c u e \in A} p(c u e \mid o b j)}{\sum_{c \in\{o b j, b g\}} p(c) \prod_{c u e \in A} p(c u e \mid c)}
\end{gathered}
$$

The posterior defined above represents the final objectness score for a window $w$. Sample windows passed from particle filter of IVT which are scored highest and lowest by the objectness measure are shown in the following Figure 3.4.

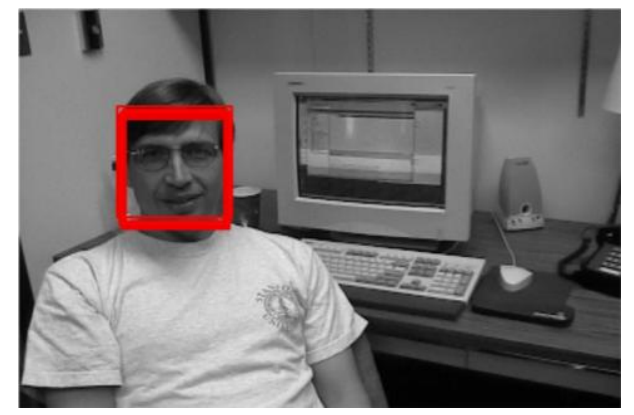

(a)

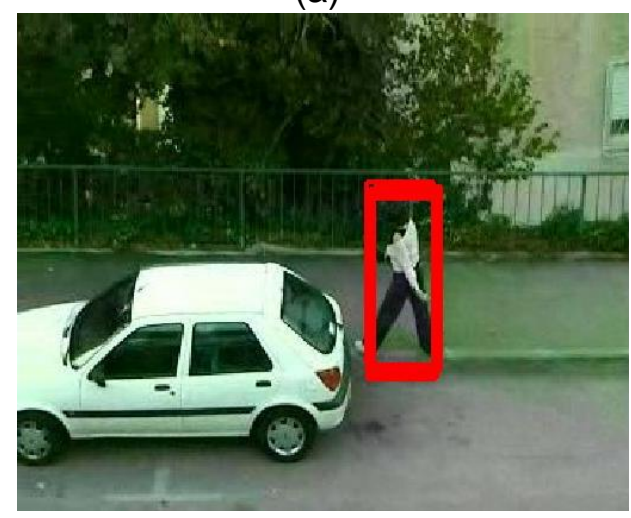

(c)

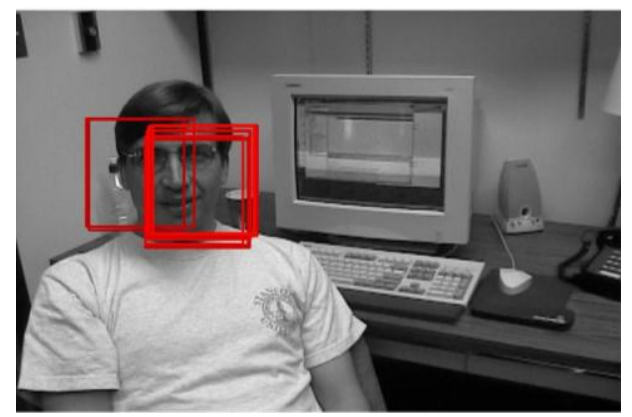

(b

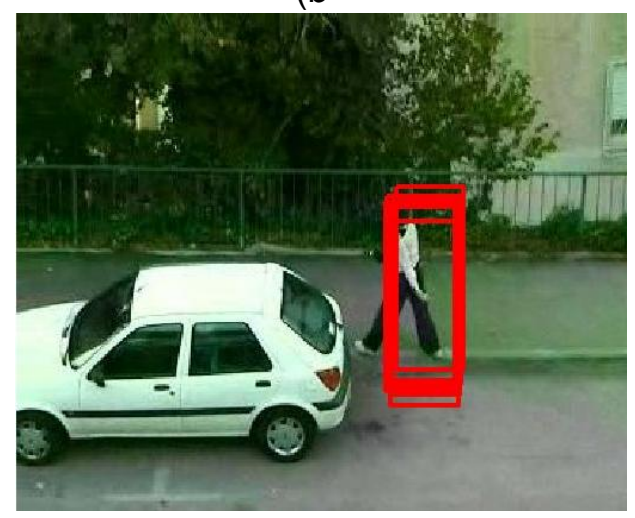

(d)

Figure 3.4 Example illustrating Objectness Measure (a) (c) Windows passed from particle filter of IVT that are scored high by objectness measure (b) (d) Windows passed from particle filter of IVT that are scored low by objectness measure 
From the Figure 3.4, it can be seen that windows fitting the object tightly are scored high while windows lying partially on the background are scored low by objectness. Thus, the windows that completely lie on the background will be scored lowest according to the objectness measure. Examples shown in the figure are frames extracted from dudek sequence obtained from Ross et.al [13] and woman sequence obtained from Adam et.al [19] which was also used for experimental evaluation in [46].

\subsection{Sequential Importance Resampling}

As mentioned before, IVT operates under condensation framework where a Gaussian dynamic model with particle filter is used to estimate the possible locations of the target in a given frame. In each frame, Sequential Importance Resampling (SIR) is performed in the particle filter based on the importance weights obtained from the likelihood computed by IVT's observation model in the previous frame. This helps in the clustering of the predictions around better previous samples which may have higher probability of containing an object thus making the distribution tighter. However, if there is problem with the likelihood measured by the observation model of the IVT, resampling around the locations predicted by the particle filter won't yield better estimates and may cause erroneous predictions of the likely states. Sequential Importance Resampling (SIR) is further discussed with an example as follows.

For instance [47], consider problem of finding a green dot and we have five samples as shown in the following figure.

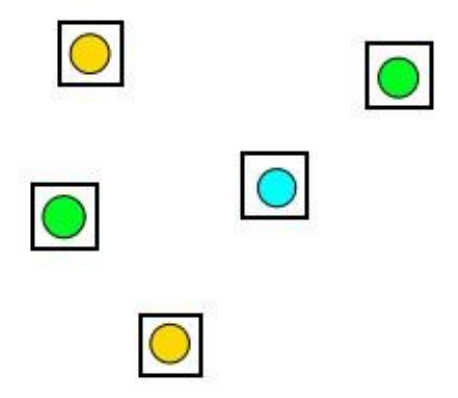


Here, the importance weight of green dots may be higher when compared to the other dots. Hence, it is better to resample around the locations of the green dots to find higher concentrations of green samples. This is performed by estimating new samples according to the cumulative distribution of weights of old samples as shown in the following figure.

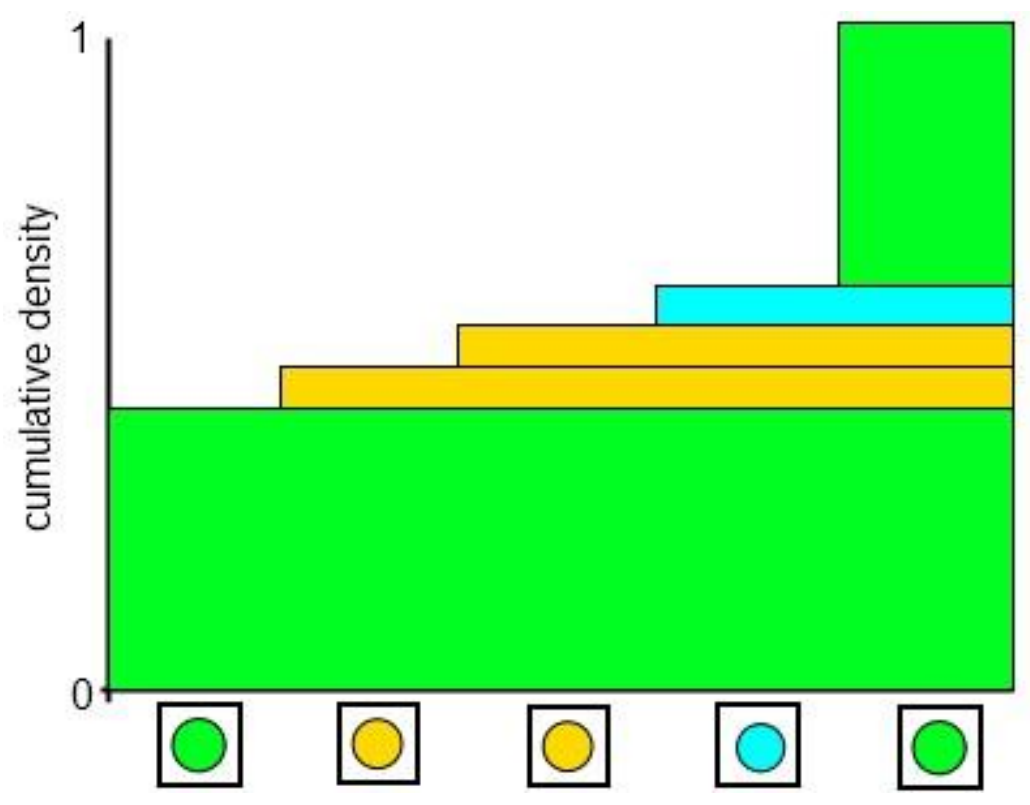

Figure 3.6 Cumulative distribution of weights. Image adopted from [47]

In the figure shown above, height of the bar represents the probability of performing resampling around that location. Resampling around higher importance weights leads to better estimates of the samples and make the distribution tighter as mentioned before [47].

Based on the above theory, we have proposed a method to detect possible problem with the observation likelihood of the Incremental learning for visual tracking algorithm. This is done by thresholding no of unique particles denoted by nup at each resampling stage of IVT. During resampling operation in the particle filter, particles are extracted based on the better previous samples which are obtained based on the cumulative distribution of likelihood weights. If the no of unique particles(nup) that are obtained at resampling stage are too high, it might indicate a problem with the likelihood of IVT. In such case, we propose to use only the likelihood computed 
by the objectness measure ignoring the likelihood computed by the observation model of the IVT. This is better depicted in the following Figures 3.7 and 3.8.
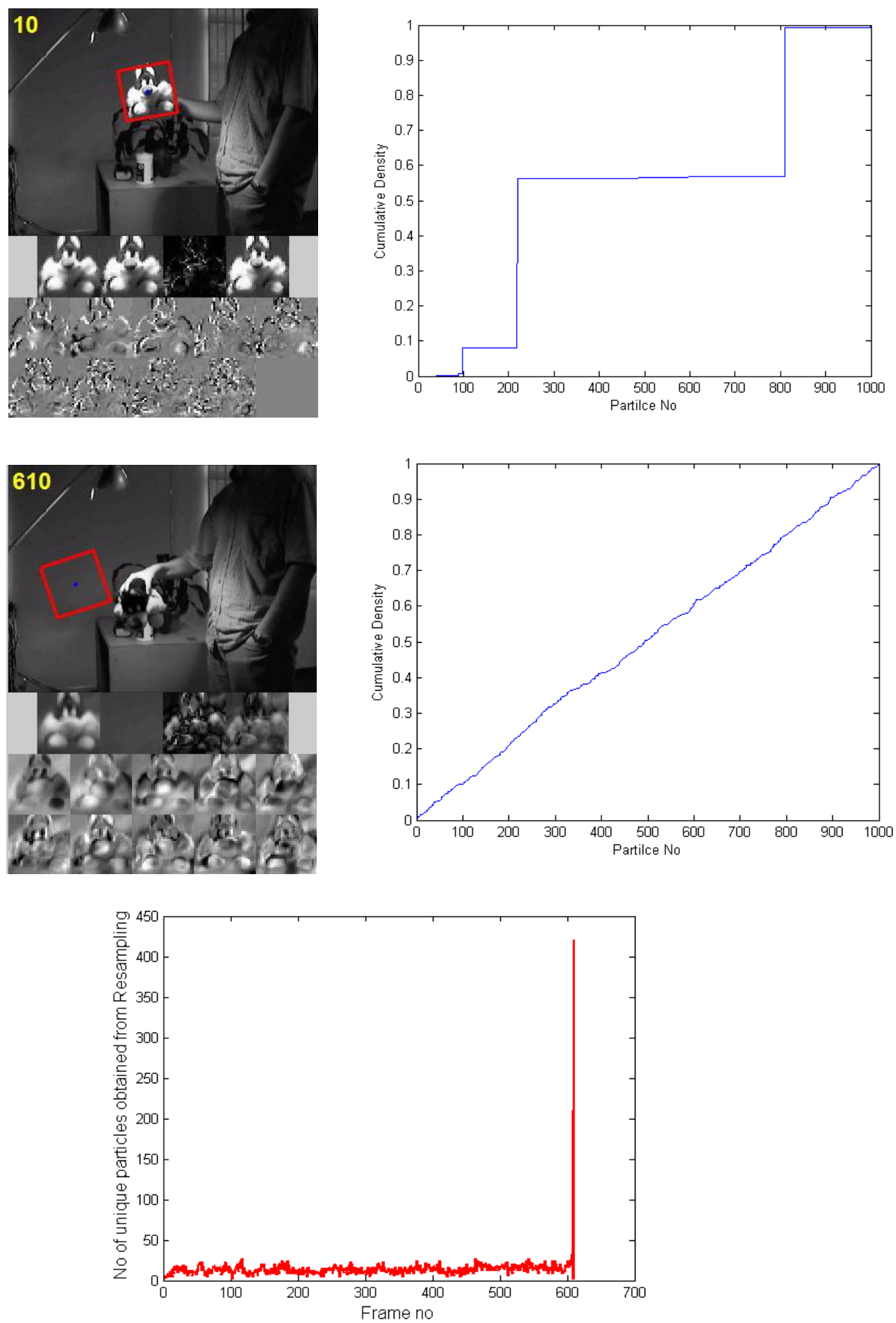

Figure 3.7 Example 1: Thresholding no of unique particles (nup) at resampling stage (a) (c) Representative frames (b) (d) Corresponding plots of cumulative densities (e) Plot showing no of unique particles at each frame. Note the abrupt increase in no of particles once tracking fails in the frame number 610 


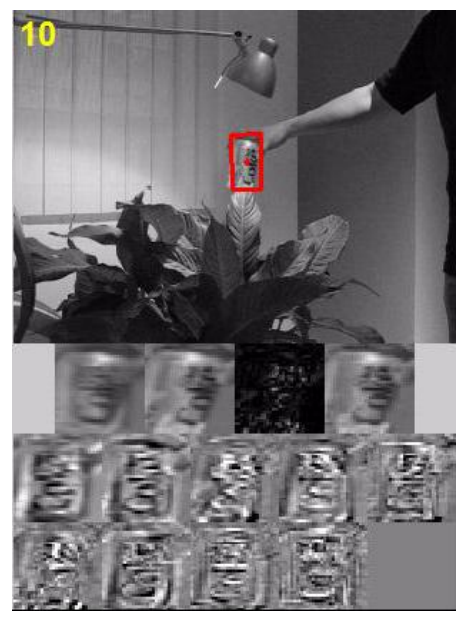

(a)

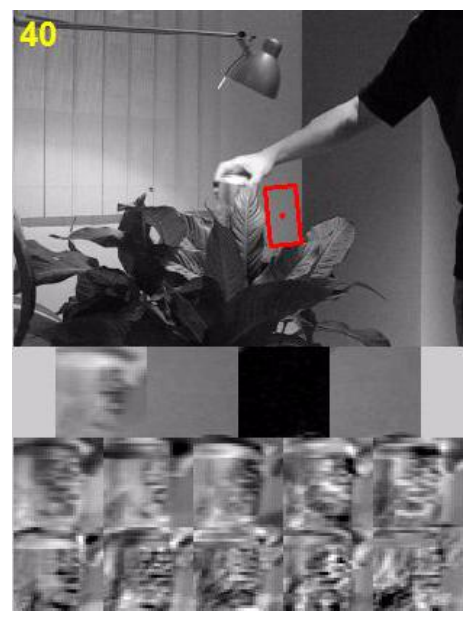

(c)

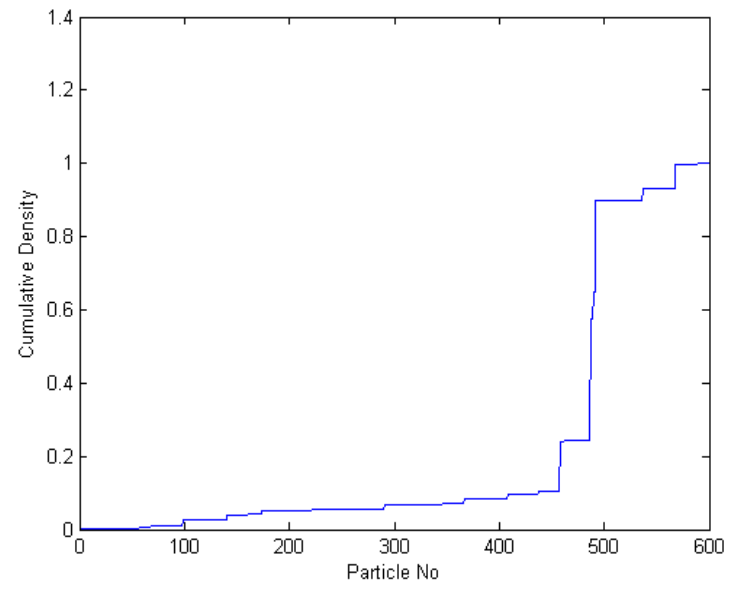

(b)

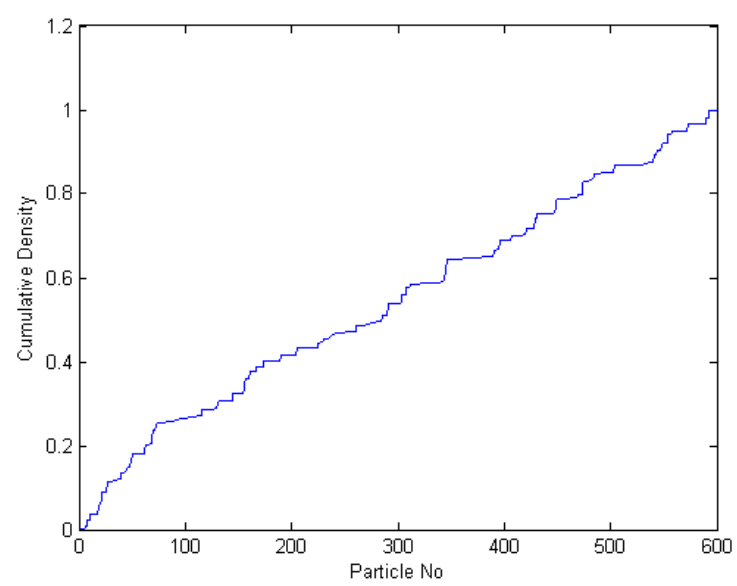

(d)

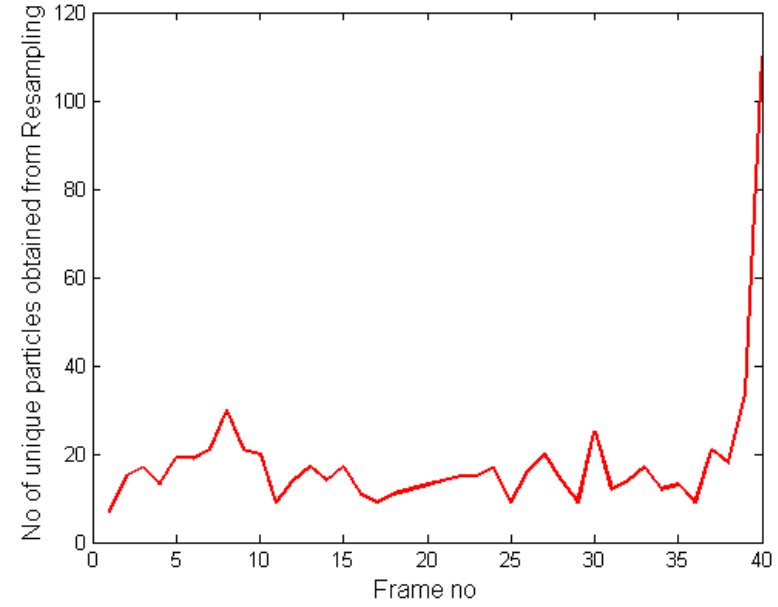

(e)

Figure 3.8 Example 2: Thresholding no of unique particles (nup) at resampling stage (a) (c) Representative frames (b) (d) Corresponding plots of cumulative densities (e) Plot showing no of unique particles at each frame. Note the abrupt increase in no of particles once tracking fails in the frame $\mathbf{4 0}$ 


\subsection{Objectness in the Context of Online Object}

\section{Tracking:}

In this work, objectness measure is used as an additional likelihood term in conjunction to the observation likelihood of IVT. As aforementioned, IVT algorithm operates under condensation framework. We use the following function to compute the final likelihood for the tracker.

Final Likelihood $=\log ($ likelihood from IVT) $+0.5 \log$ (likelihood from objectness $)$

Here, 0.5 and 1 are weights for likelihood obtained from objectness measure and observation likelihood of IVT respectively which are chosen empirically.

The image window corresponding to the maximum likelihood calculated from equation (1) is stored for the update of appearance model.

In the cases where observation likelihood of IVT is very low, only likelihood of objectness measure is considered for the update of appearance model. As discussed in the section 3.2, this condition can be detected by thresholding the no of unique particles (denoted nup) obtained at each resampling step in the condensation algorithm. If the no of unique particles obtained from resampling step is greater than certain threshold, it may indicate a problem with the likelihood obtained from IVT observation model. In such cases, only likelihood from objectness is used to update the template. Also, the tracker is reinitialized at times when the likelihood of IVT is low, but the likelihood of image window containing the object (according to the objectness measure) is higher. This way, the past erroneous accumulations in the template update can be cleared thus ensuring the reliability of tracking.

\subsubsection{Tracking Failure Detection}

Every tracker, no matter how robustly designed will fail in a particular scenario. Detecting tracking failure like complete drift of the tracker from target to the background can be useful for taking automated measures to recover from failures. Automated measures may range from simple template matching to learning based schemes like object detection to reacquire the target. Also depending on the application, measures can be interactive and semi-automated making user aware of the tracking failure and allowing him to take recovery measures such as 
reinitializing the tracker etc. This might help in improving overall tracking reliability. Some of the novel tracking failure detection methods based on forward-backward tracking error and reversible nature of tracker under particle filtering framework are discussed in [48] and [49] respectively. In the proposed approach by thresholding both no of unique particles at resampling stage of IVT and the sum of scores of top hundred windows from objectness, we can possibly detect a condition in which IVT has completely drifted to the background as shown

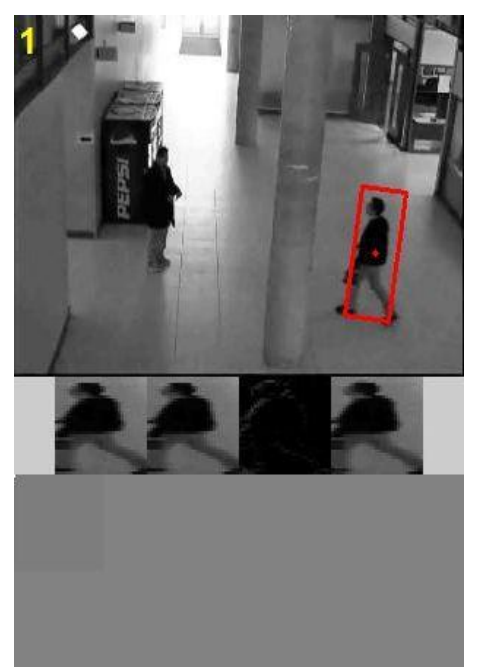

(a)

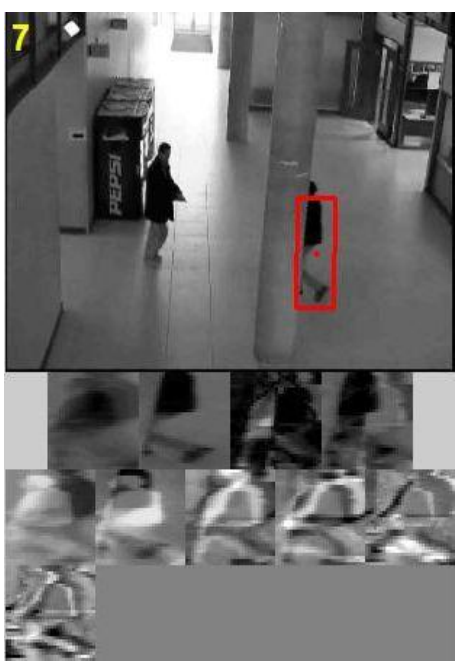

(b)

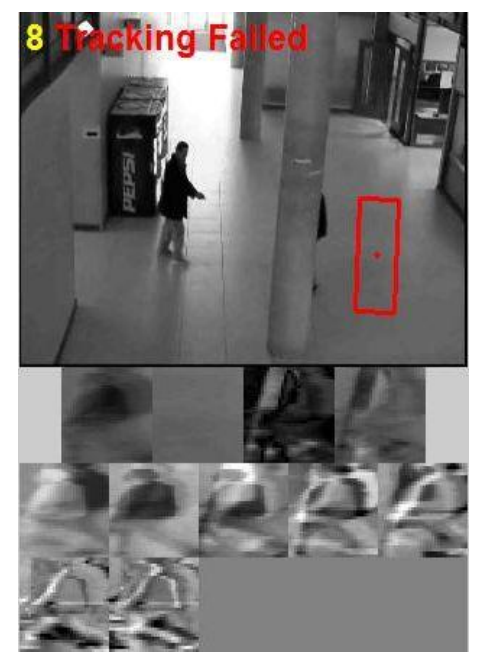

(c)

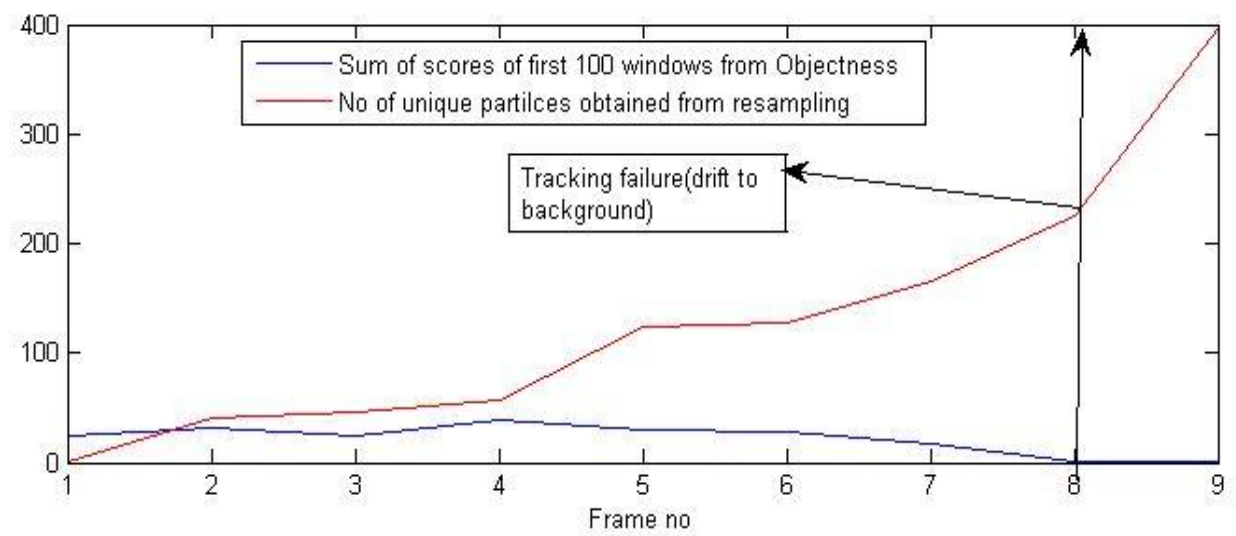

(d)

Figure 3.9 Implicit Tracking Failure Detection (a) (b) (c) Representative frames from pop machines sequence [26] (d) Plot showing the thresholding to detect tracking failure

in the Figure 3.9. In the next section we present summary of the proposed algorithm. 


\subsection{Summary of Proposed Algorithm}

Initialization: Initialize parameters for IVT algorithm. Parameters for computing objectness are mostly fixed. Thresholds for detecting the condition of using only objectness is adjusted based on the sequence. Target position is given manually in the first frame. A single particle is used to indicate this location.

\section{Tracking:}

1. Initialize the Eigen basis to be empty and mean $\mu$ of the appearance model to the target patch in the first frame. Initiate flag=0(Tracking failure flag)

2. Proceed to the next frame. Compute the no of unique particles (denoted (nup)) from resampling algorithm based on cumulative distribution of weights from likelihood of last frame.

3.if (nup>predefined threshold)

Do not resample

else

Resample and extract the particles.

endif

Draw the particles from the particle filter based on dynamic model.

4. For each particle, extract the image window from the current frame and compute the observation likelihood of IVT and likelihood according to objectness measure. Calculate the combined likelihood using equation (1).

5. Compute nup again at this step.

if (nup<predefined threshold)

Use combined likelihood computed from equation (1)

else

Use only likelihood from objectness measure.

endif

6. Store the image window corresponding to the most likely particle (maximum likelihood) and after accumulating the desired no of images, update appearance model of IVT.

7. if (nup>predefined threshold \&\& sum of likelihoods of first hundred particles from objectness measure $<$ certain threshold)

Tracker drifted to background. Assign flag equals to 1 .

endif

if (nup>predefined threshold \&\& likelihood from objectness>certain threshold $\& \&$ flag $==0$ ) endif

Reinitialize the tracker with current location (from objectness measure)

8. Go to step 2.

Figure 3.10 Summary of the proposed algorithm 
Summary of modified IVT algorithm after incorporating objectness is shown in the above Figure 3.10. The block diagram of the proposed tracking system is shown in the Figure 3.11.

For illustrative purposes, images at different stages of the proposed approach as shown in the block diagram (Figure 3.11) are depicted in the Figure 3.12 on basketball sequence obtained from [36].

Here, particle filter of IVT computes the possible locations of the object in the current frame based on the likelihood of the IVT observation model from the previous frame. These image windows possibly containing the object (shown as blue windows in the Figure 3.12(b)) were scored by both observation model of IVT and the objectness measure. Red windows in the images in the Figure 3.12,(c) and (d) shows the top ten windows which are scored highest by IVT and objectness respectively while windows represented by black color denotes the lowest scores by respective models. As mentioned before both the scores are combined in log likelihood fashion (with respective weights) and maximum of combined score gives the object location in the subsequent frame as shown in the Figure $3.12(\mathrm{e})$.

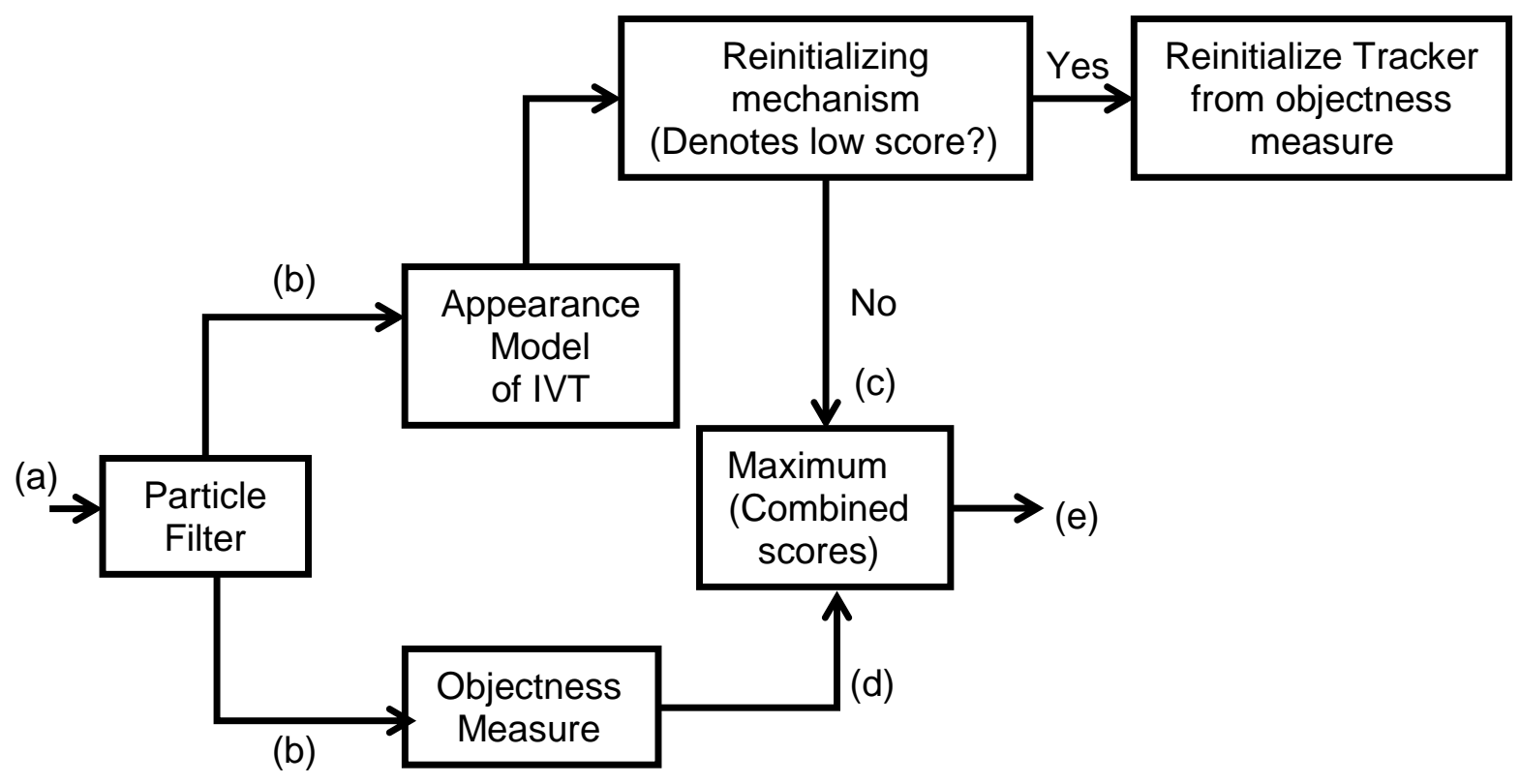

Figure 3.11 Block diagram of proposed tracking system 


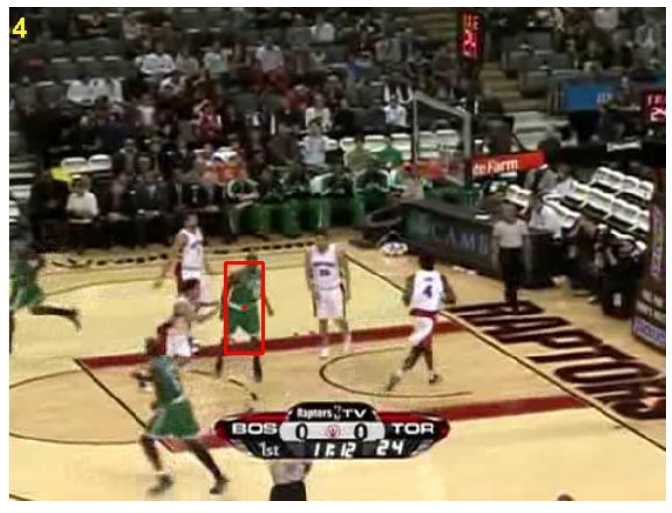

(a)

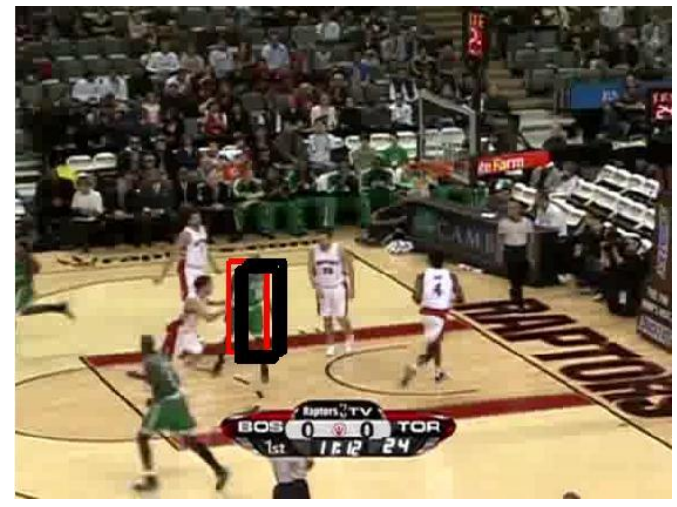

(c)

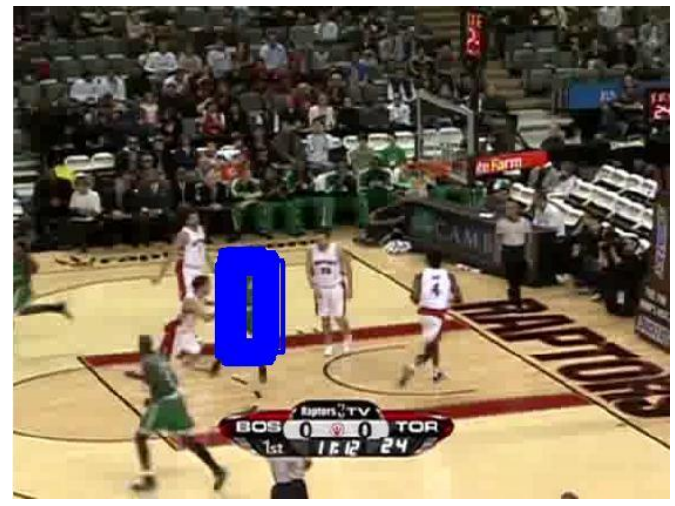

(b)

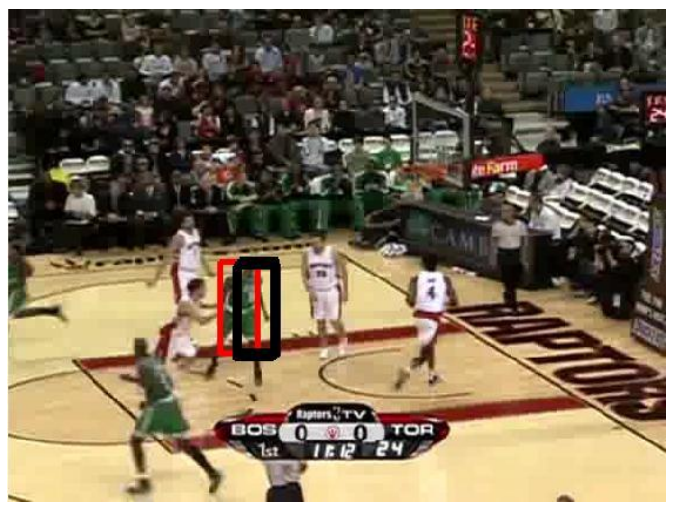

(d)

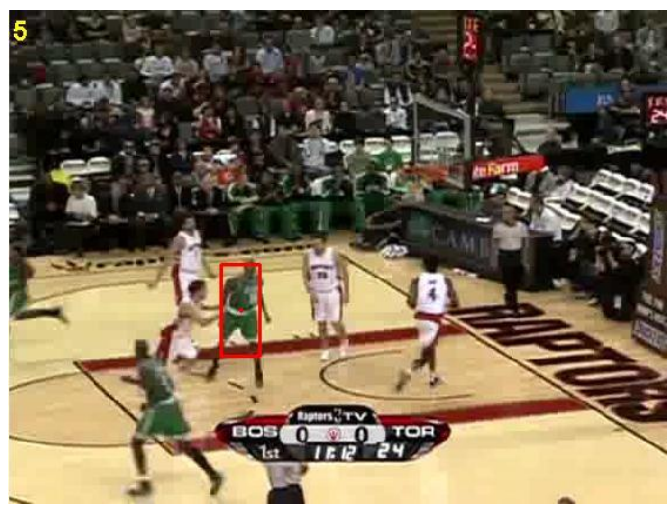

(e)

Figure 3.12 Proposed tracking system: Illustrative frames (a) Previous frame (b) Output from particle filter (c) output from IVT (d) output from objectness measure (e) Estimated object location in the current frame

In the event of possible problem with the IVT's observation model, score from objectness measure is used to determine the location of the object. Also if we are confident that objectness measure does locate the target in the given frame (which can be done by thresholding the maximum score returned by the objectness measure module as outlined in the 
summary of the proposed approach), the tracker is reinitiated with its current location as the input. This may help in getting rid of the previous erroneous accumulations in the appearance model of the tracker thus ensuring the tracking reliability. In the next chapter we discuss both the qualitative and quantitative results of the proposed method. 


\section{Experimental Results}

In this chapter we discuss both qualitative and quantitative results of the proposed approach on different video sequences. It is critical to evaluate the performance of any tracking algorithm on a representative dataset which include different tracking challenges and scenarios. Although there exists several datasets made publicly available for surveillance scenarios such as PETS, CAVIAR [50] and VIVID [51]databases, these are more specific to certain objects like cars and persons and often assume a static background [52]. Hence to the best of our knowledge there is no standard database that is specifically formed for evaluating general object tracking algorithms. More recently, different video sequences incorporating variety of challenges and tracking scenarios are made publicly available by researchers on object tracking in their respective works [19] [35] [18] [36] [13] [53] [54] [55] [15]. We have collected large number of these sequences and formed a dataset which varies greatly in terms of the type of the object, scene conditions from one sequence to another and incorporates challenging scenarios such as occlusion (full and partial), pose and scale changes of the target etc.

We have evaluated Incremental Visual Tracker (IVT) [13] on each sequence of the representative dataset formed and collected a database of 10 video sequences in which IVT drifts to the background rather than tracking the target as a result of different challenges posed during the tracking. Sequences used for experimental evaluation are listed in the Table 4.1 along with the challenging factors in them and the sources from which these sequences are obtained or extracted. We evaluated proposed approach of incorporating objectness in to IVT on these sequences. The experimental results show that proposed method does help to alleviate its drift to background problem on certain challenging sequences.

We discuss the experimental setup here. The base codes for IVT and objectness are obtained from websites of respective authors which are made publicly available for research purposes. The parameters for each sequence (number of particles, initialization of the target and other parameters like variances of the dynamic model, forgetting factor of IVT) are tuned accordingly and kept same while evaluating IVT and the proposed approach. As many other tracking approaches in the literature, initial bounding box of the target in the first frame of the video sequence is provided manually. This is depicted in the Figure 4.1. Alternatively an object detector (although involves more complexity) can be employed to detect the target object in the 
first frame. Parameters for objectness measure are mostly fixed. Thresholds for detecting the tracking failure and reinitialization are kept mostly same (hard thresholds) for each sequence. We present qualitative comparison of the sequences in which we have observed the improvement in the tracking performance of the proposed approach over the IVT in the next section.

Table 4.1 Sequences used and challenging factors along with their sources

\begin{tabular}{|c|c|c|c|}
\hline Sequence & \#Frames & Challenging factors & Source \\
\hline Sylvester & 1344 & $\begin{array}{c}\text { Illumination conditions, fast motion of the } \\
\text { object, in plane/out of plane rotation }\end{array}$ & $\begin{array}{c}\text { Ross et.al } \\
\text { [13] }\end{array}$ \\
\hline Bird1 & 147 & $\begin{array}{c}\text { Fast motion ,smaller size of the object, } \\
\text { similar objects in the scene }\end{array}$ & $\begin{array}{c}\text { Wang et.al } \\
\text { [36] }\end{array}$ \\
\hline Bird2 & 99 & Occlusion, smaller size of the object & $\begin{array}{c}\text { Wang et.al } \\
\text { [36] }\end{array}$ \\
\hline Basketball & 401 & Nonlinear deformation, occlusion, fast & $\begin{array}{c}\text { Wang et.al } \\
\text { [36] }\end{array}$ \\
\hline Transformer & 124 & Non rigid deformation & $\begin{array}{c}\text { Kwon et.al } \\
\text { [56] }\end{array}$ \\
\hline Coke Can & 292 & Occlusion, fast motion & $\begin{array}{c}\text { Babenko et.al } \\
\text { [35] }\end{array}$ \\
\hline Pop Machines & 37 & Occlusion, similar objects in the scene & $\begin{array}{c}\text { Cannons } \\
\text { et.al [26] }\end{array}$ \\
\hline Tiger 1 & 354 & Fast motion of the object, background \\
clutter, occlusion & $\begin{array}{c}\text { Babenko et.al } \\
\text { [35] }\end{array}$ \\
\hline Girl & 258 & $\begin{array}{c}\text { Prolonged occlusion } \\
\text { [36] }\end{array}$ \\
\hline [19]
\end{tabular}




\subsection{Qualitative Assessment}

Figures 4.2 to 4.5 shows the qualitative comparison of the IVT and proposed approach on certain sequences where the proposed method partially alleviated the complete drift to background problem of the IVT. 

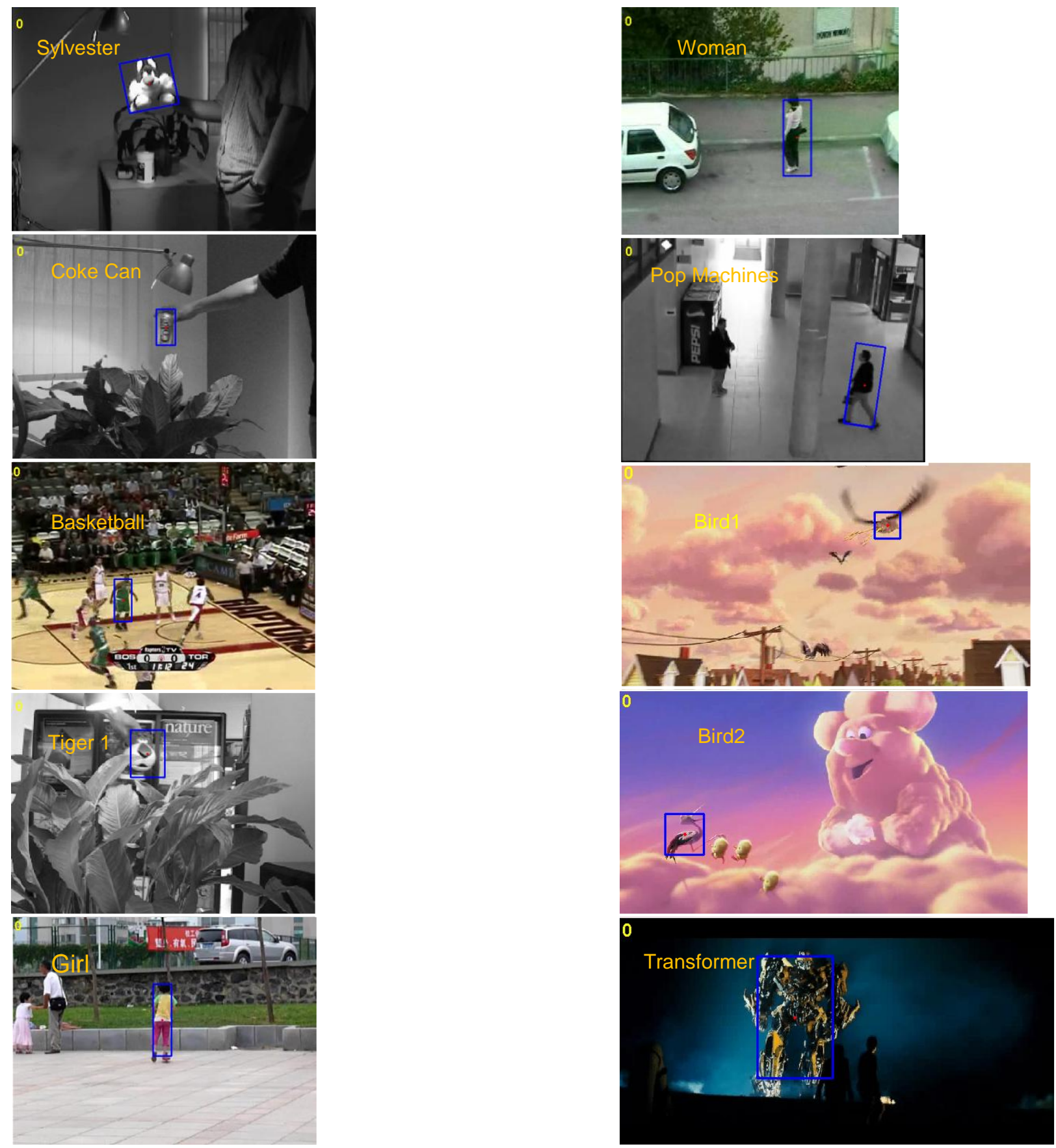

Figure 4.1 First frames of the tracking video sequences used for experimental evaluation. Initial bounding box of the target is supplied manually (Represented by blue bounding box where red dot denotes center of the box). 

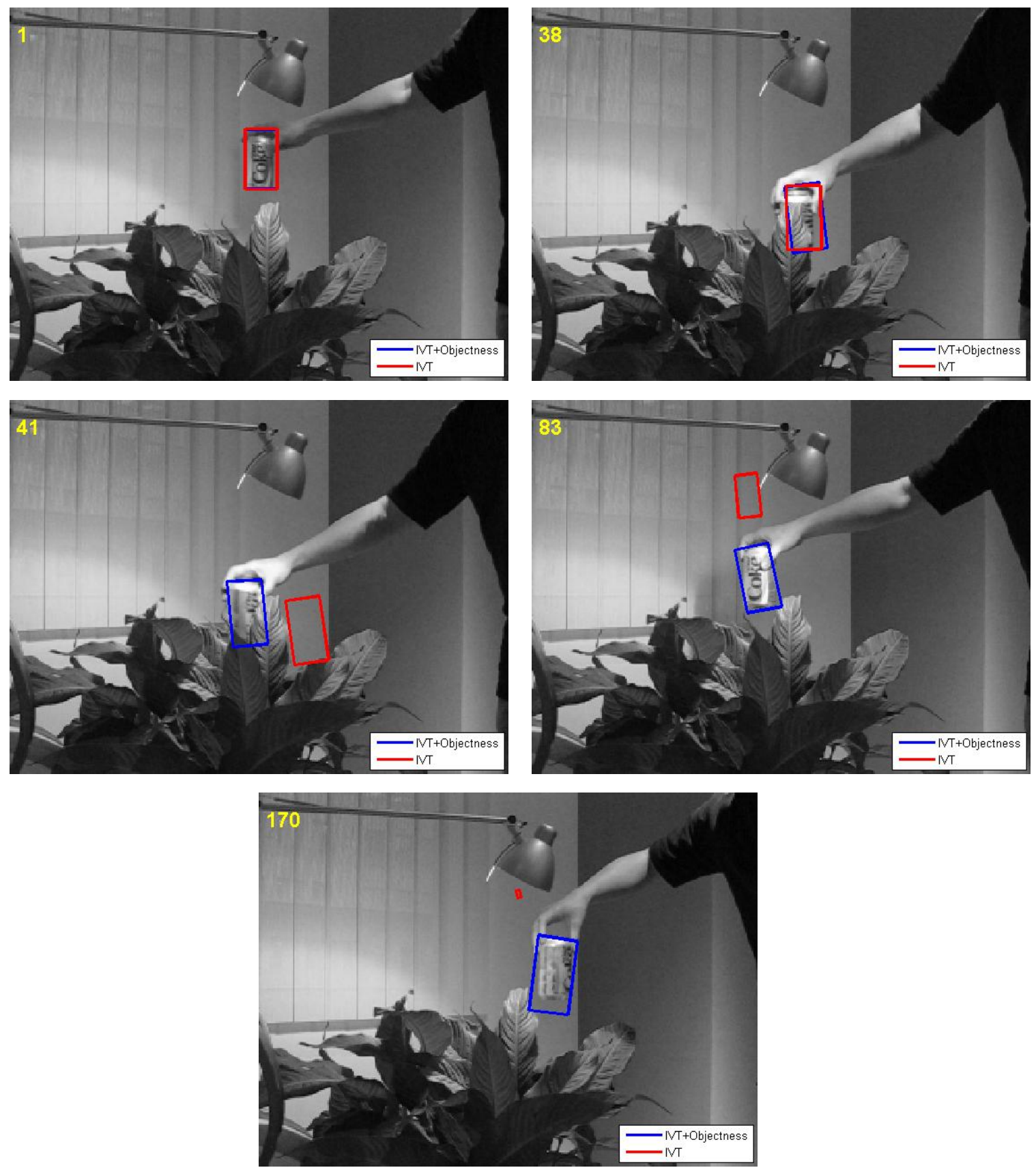

Figure 4.2 Qualitative Assessment: Representative Frames: Coke Can sequence. IVT represented by red rectangle and tracking by proposed approach denoted by blue rectangle. Frame number is denoted in the top left corner of the image. Note that when occlusion occurs in the 38th frame,IVT completely drifts to background while proposed approach is able to recover from occlusion as a result of reinitialization. 

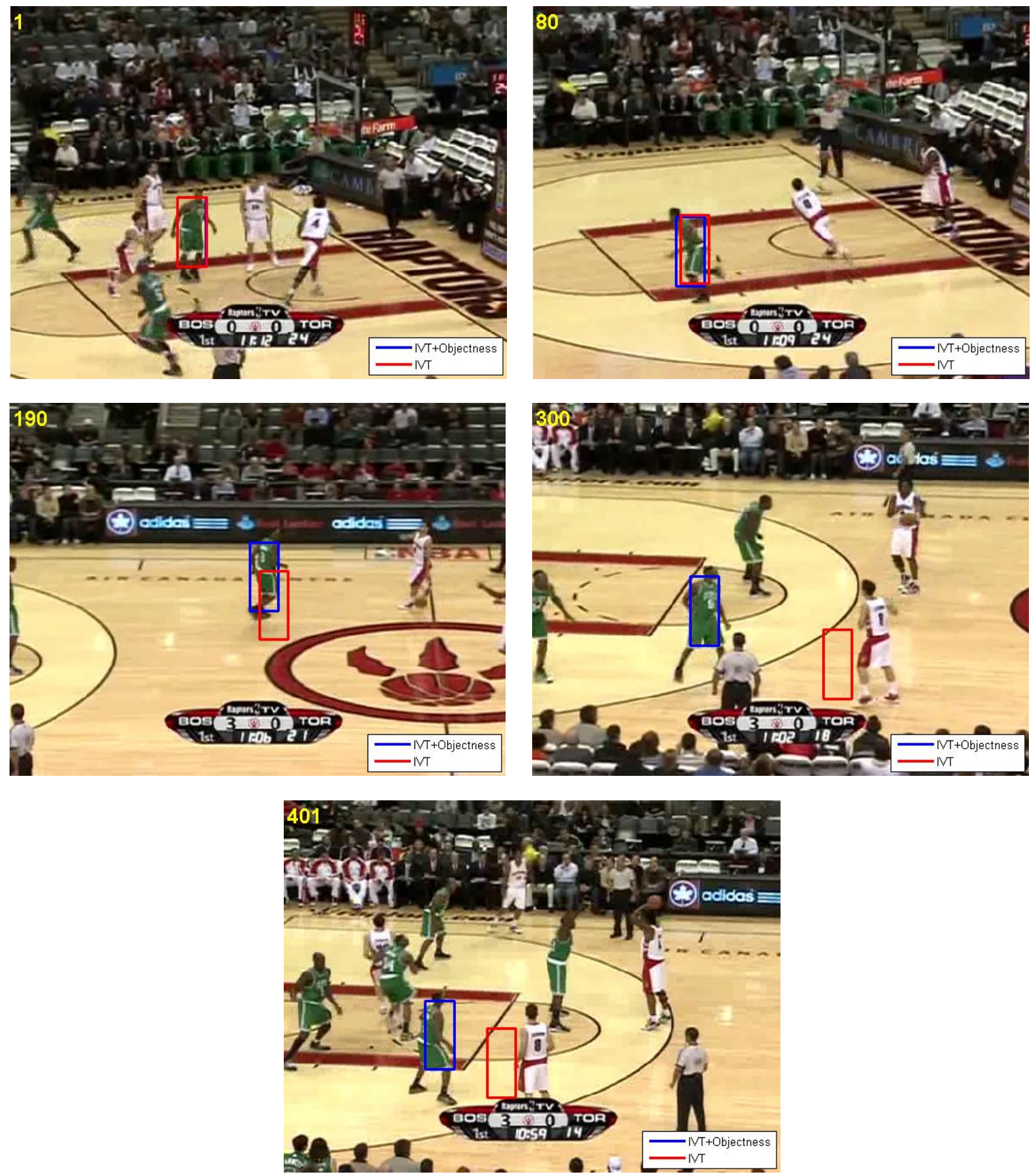

Figure 4.3 Qualitative Assessment: Representative Frames: Basketball sequence. IVT represented by red rectangle and tracking by proposed approach denoted by blue rectangle. Frame number is denoted in the top left corner of the image . Note that due to heavy shape deformation of the player,IVT drifts to background while proposed approach is able to track him successfully. 

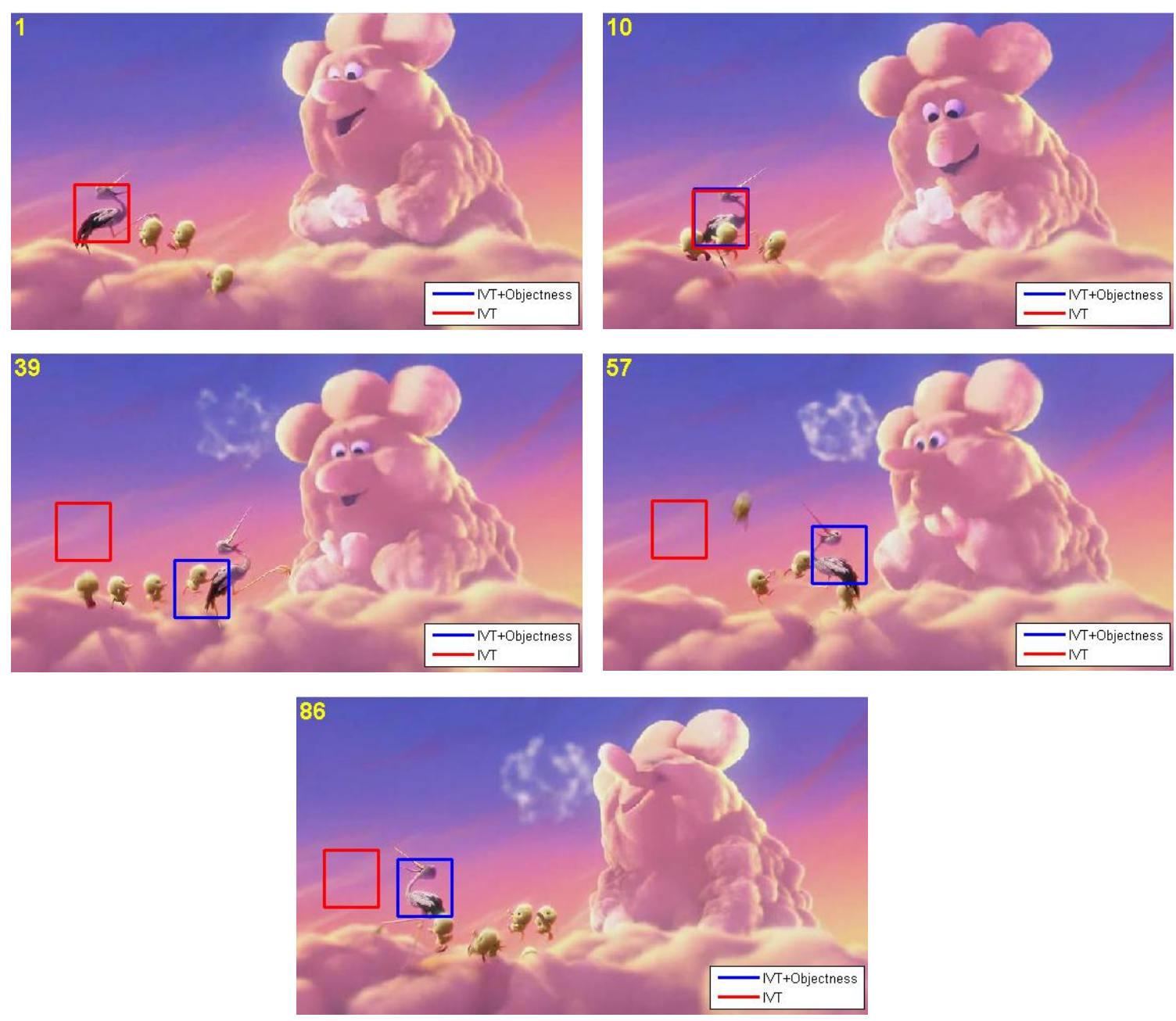

Figure 4.4 Qualitative Assessment: Representative Frames: Bird 2 sequence. IVT represented by red rectangle and tracking by proposed approach denoted by blue rectangle. Frame number is denoted in the top left corner of the image. Note that due to heavy occlusion of the target in $10^{\text {th }}$ frame ,IVT has completely drifted towards the background. On the other hand,proposed approach although drifts to background initially, due to reinialization in the later frames, was able to recover from the drift. 

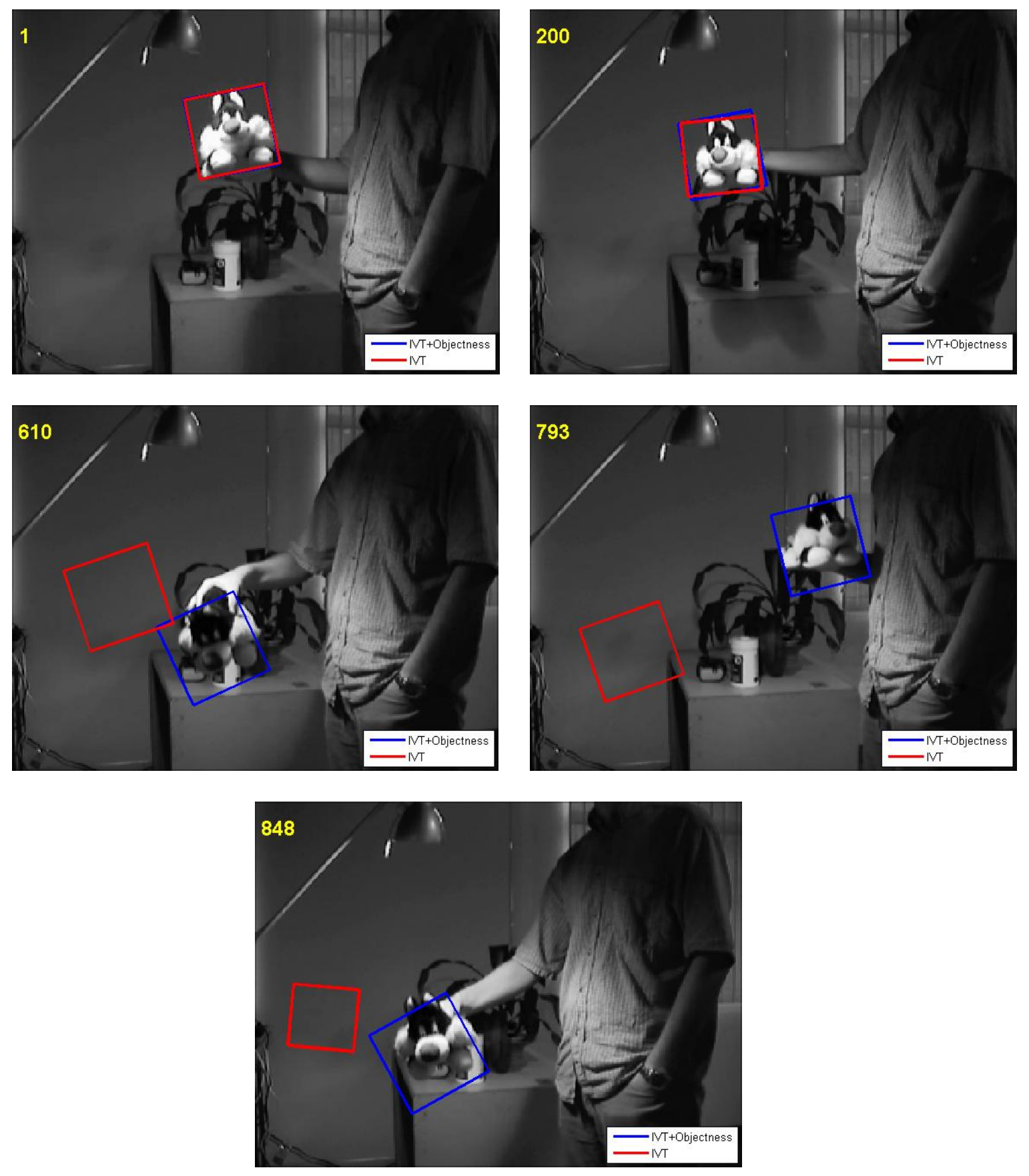

Figure 4.5 Qualitative Assessment: Representative Frames: Sylvester sequence. IVT represented by red rectangle and tracking by proposed approach denoted by blue rectangle. Frame number is denoted in the top left corner of the image. Note that as a result of extreme pose changes of the object,IVT has completely drifted to background,while proposed approach is able to track the target through the challenges. 


\subsection{Quantitative Assessment}

In this section, we provide quantitative analysis of the tracking performance of both IVT and proposed approach on the four challenging sequences in which we have observed the performance enhancement via proposed method. To compute quantitative performance, the region of overlap between the tracker output and ground truth rectangle is measured by using following relation which give the tracking success score for a frame. This is graphically represented in the following Figure 4.6.

$$
\text { Score }=\frac{\operatorname{area}\left(R O I_{T} \cap R O I_{G}\right)}{\operatorname{area}\left(R_{O} I_{T} \cup R O I_{G}\right)}
$$

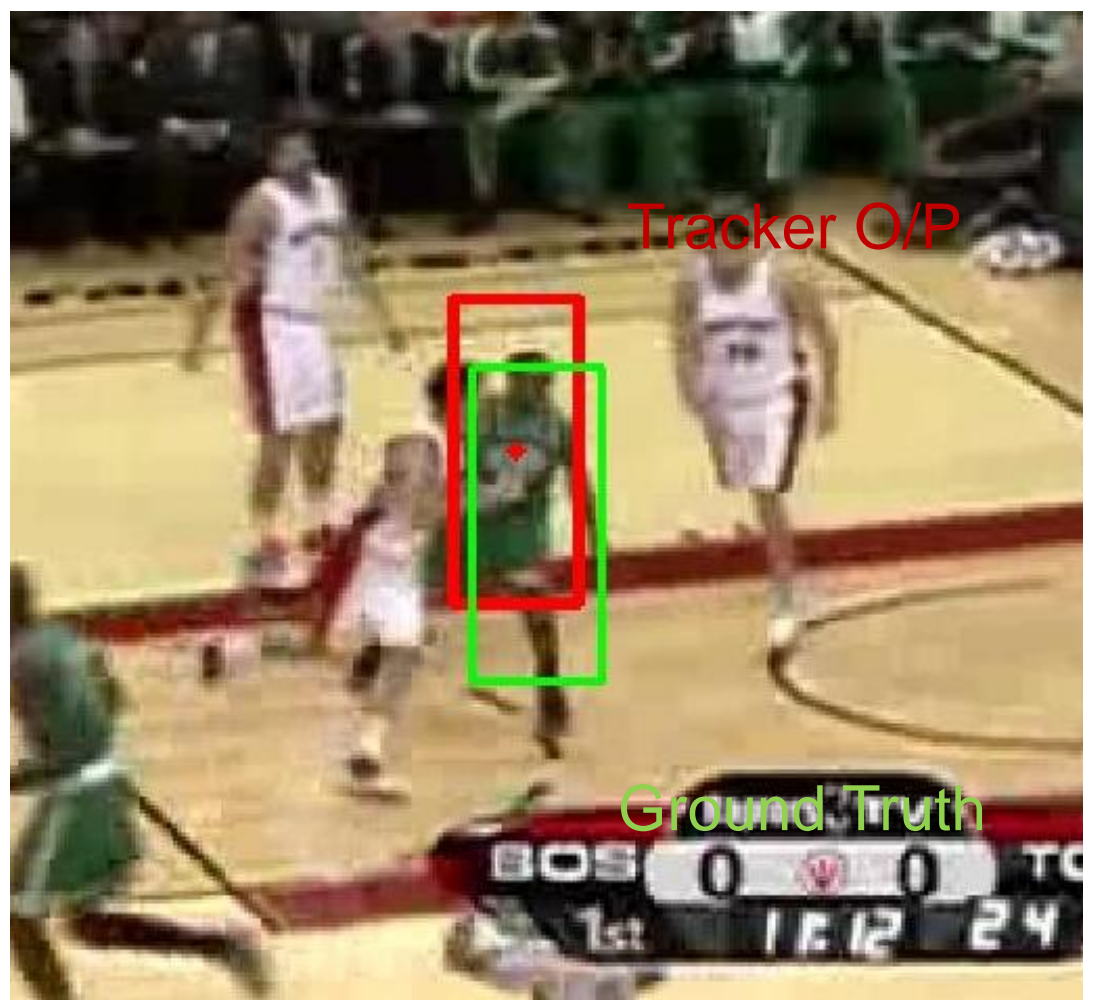

Figure 4.6 Computation of tracking success score: Red rectangle denotes tracker output and green rectangle denotes the ground truth. Success score is calculated by the relation given above 
Success score is computed for each individual frame in the video sequence. Plots of frame number vs. tracking score for four sequences for both IVT and proposed approach are provided below.

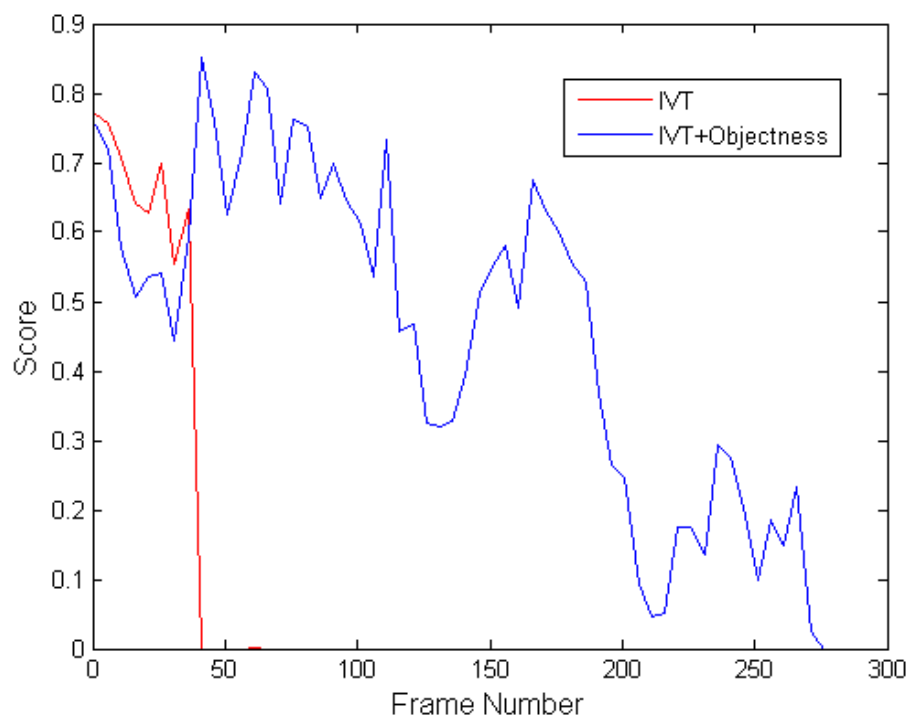

Figure 4.7 Quantitative Assessment: Coke Can Sequence: Frame number VS Tracking score. A frame which has a score above $1 / 3$ can be considered a success, otherwise a miss.

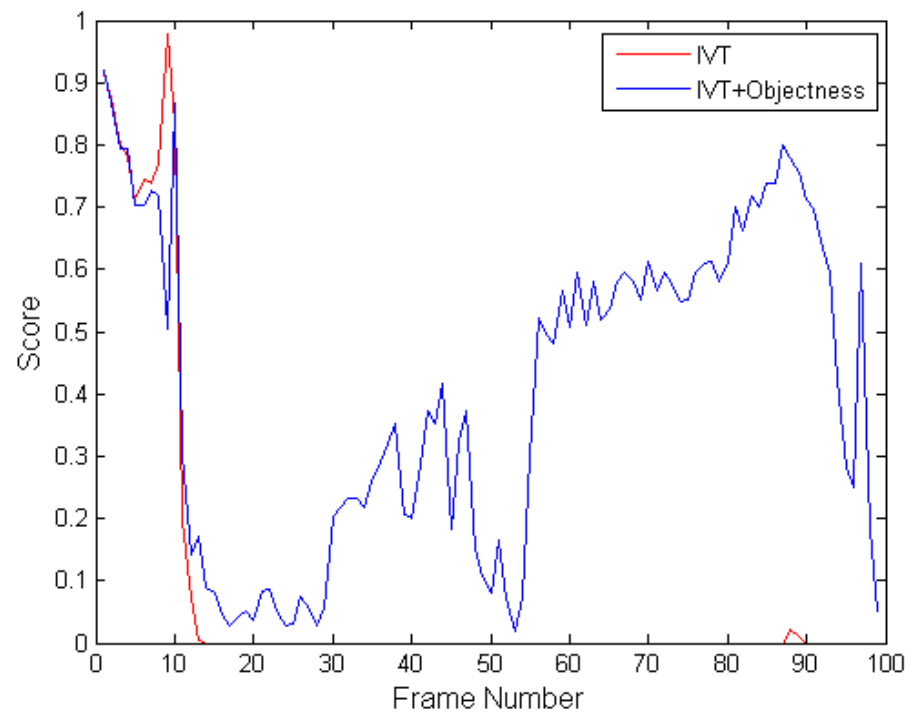

Figure 4.8 Quantitative Assessment: Bird 2 Sequence: Frame number VS Tracking score. A frame which has a score above $1 / 3$ can be considered a success, otherwise a miss. 


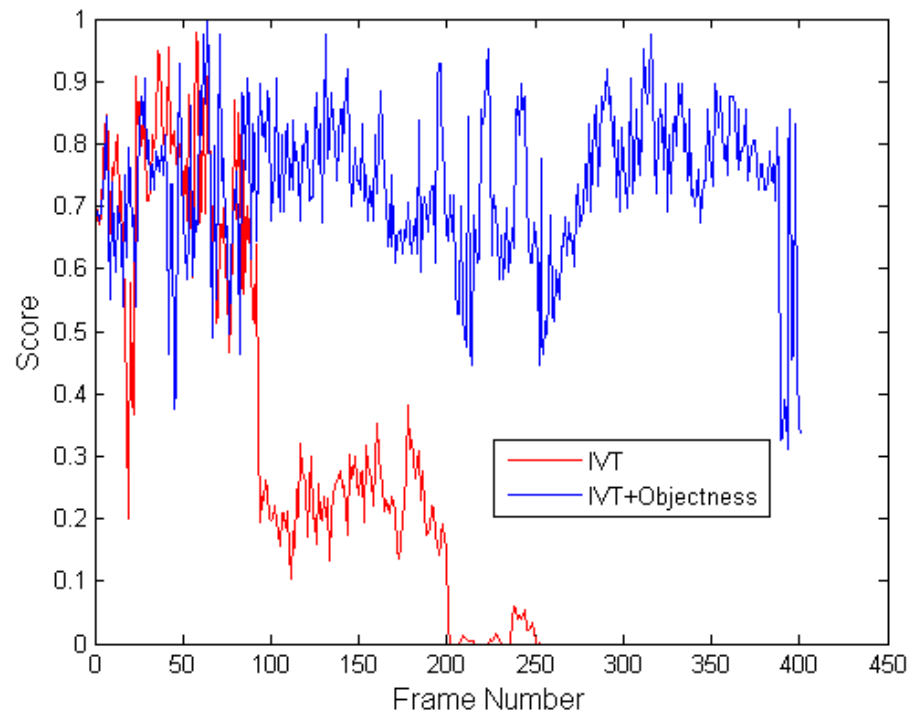

Figure 4.9 Quantitative Assessment: Basketball Sequence: Frame number VS Tracking score. A frame which has a score above $1 / 3$ can be considered a success, otherwise a miss.

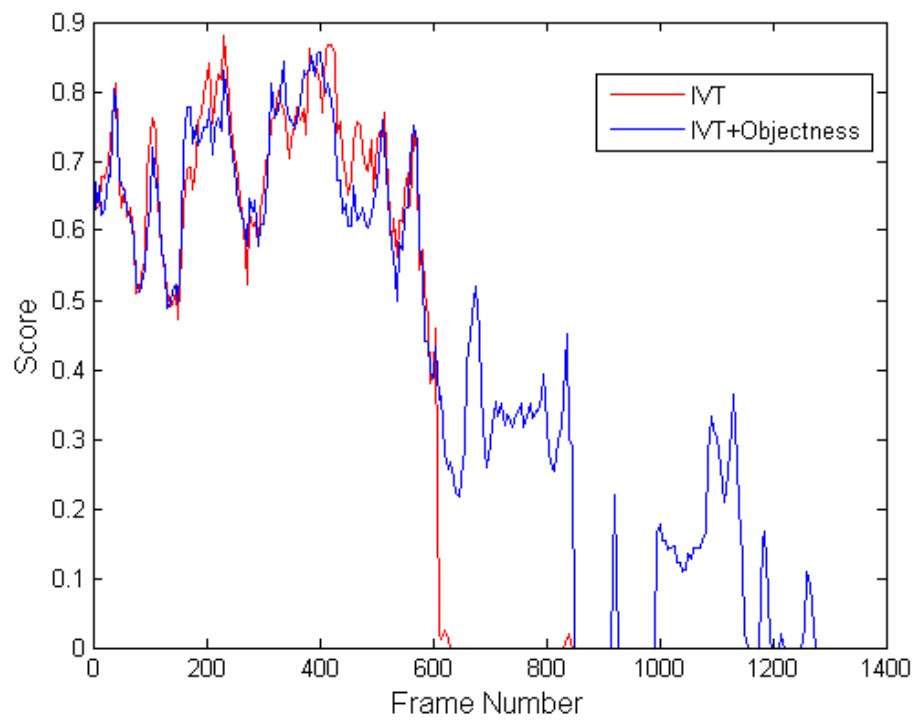

Figure 4.10 Quantitative Assessment: Sylvester Sequence: Frame number VS Tracking score. A frame which has a score above $1 / 3$ can be considered a success, otherwise a miss. 
For comparative purposes, tracking score above 1/3 can be considered as success for a particular frame and below this can be considered as a miss [17]. Success rate is calculated by dividing no of frames in which tracking is successful by the total no of frames in the sequence. Success rate computed in this way is provided for both IVT and proposed method for four sequences in Table 4.2.Ground truth for coke can and sylvester sequences are obtained from Babenko et.al [7] which has location of the target marked for every $5^{\text {th }}$ frame. Ground truth for bird 2 and basketball sequence are obtained from Wang et.al [36] where location of the target is annotated every frame. From the above plots, it can be observed that occasionally IVT has higher score than the proposed method for certain frames due to the small inconsistencies introduced while combining the IVT and objectness. This can also be due to the rectangular window assumption of objectness while IVT assumes affine motion.

Table 4.2 Quantitative Comparison: Success Rates of IVT and proposed method

\begin{tabular}{|c|c|c|c|}
\hline Sequence & \# Frames & Success rate of IVT & $\begin{array}{c}\text { Success rate of } \\
\text { IVT+Objectness }\end{array}$ \\
\hline Coke Can & 292 & 13.70 & 61.64 \\
\hline Bird 2 & 99 & 10.10 & 55.56 \\
\hline Basketball & 401 & 23.44 & 99.5 \\
\hline Sylvester & 1344 & 45.39 & 55.06 \\
\hline
\end{tabular}

From the plots, it can be observed that IVT completely fails (success score of zero) early in the video to track the object as a result of occlusion in frame numbers 38 and 10 of coke can and bird 2 sequences respectively while proposed approach is able to cope up with this challenge to certain extent. In the basketball sequence, due to heavy shape deformation (nonlinear) of the player, IVT drifts completely towards the background while proposed approach is able to track the player successfully throughout the sequence(we have used a part of the basketball sequence obtained from Wang et.al [36] for our evaluation containing 401 frames to avoid memory limitation problem in MATLAB). The success of proposed approach on 
this sequence can be attributed to the series of reinitializations of the tracker through the shape deformation of the object (basketball player). Finally, in the Sylvester sequence, IVT completely drifts to background in the frame 610 as a result of combination of extreme pose change of the object and illumination conditions. In this sequence, due to reinitialization of the tracker in the proposed approach it is able to track the object till $848^{\text {th }}$ frame where it fails due to out of plane rotations of the object.

Proposed approach on an Intel 2.8 GHz PC with 3GB memory runs about 2 frames/sec on a video sequence of resolution 320X240(In MATLAB) with 600 particles. On the other hand, IVT runs about 10 frames/sec under same conditions. Additional overhead in the proposed approach is due to the computation of likelihood with both the cues Color Contrast (CC) and Superpixel Straddling (SS). However, the code is currently not optimized and can be improved to increase the efficiency.

\subsection{Comparison with Other Online Tracking}

\section{Algorithms}

Here we present the quantitative comparison of few other tracking algorithms in the literature which employ online paradigm for appearance models. We have compared four different trackers along with the IVT and proposed method for the ten sequences in the experimental dataset. The trackers compared are Boosting Tracker [20], SemiBoosting Tracker [21], BeyondSemiBoosting Tracker [22] and I1-tracker [15]. All the trackers are supplied with same initial bounding box (IVT and proposed method may involve slight rotation of the initial bounding box as the motion model used is affine transform). L1-tracker is particle filter based, hence we have used same number of particles employed for each sequence while evaluating IVT and proposed method and other parameters were kept unchanged as provided by the authors(We have used source code made available by the authors of I1-tracker for empirical evaluation). For remaining three trackers i.e. Boosting tracker, SemiBoost tracker and BeyondSemiBoost tracker we have used executable binaries (precompiled) with tunable parameters made available by their respective author's. These trackers work under online boosting framework. We have used slightly better parameters than the default parameters provided by the authors in the binaries (Based on the description of the parameters). It is important to note that Boosting Tracker while evaluated using its executable file will stop the 
tracking once classifier threshold falls below certain threshold. Hence it may be still possible to obtain better results with the source code and more tunable parameters. Also, SemiBoosting tracker and BeyondSemiBoosting tracker are not included in the comparison of the performance in sylvester sequence since ground truth for this sequence, is only available every $5^{\text {th }}$ frame and these trackers gave intermittent detections. For the same reason, SemiBoosting tracker is not compared for coke can sequence. Comparison shows that the proposed method performed favorably against the other trackers on these sequences. Table 4.3 illustrates the comparison between the trackers on all the sequences including those in which improvement in performance over IVT is observed via proposed method. 


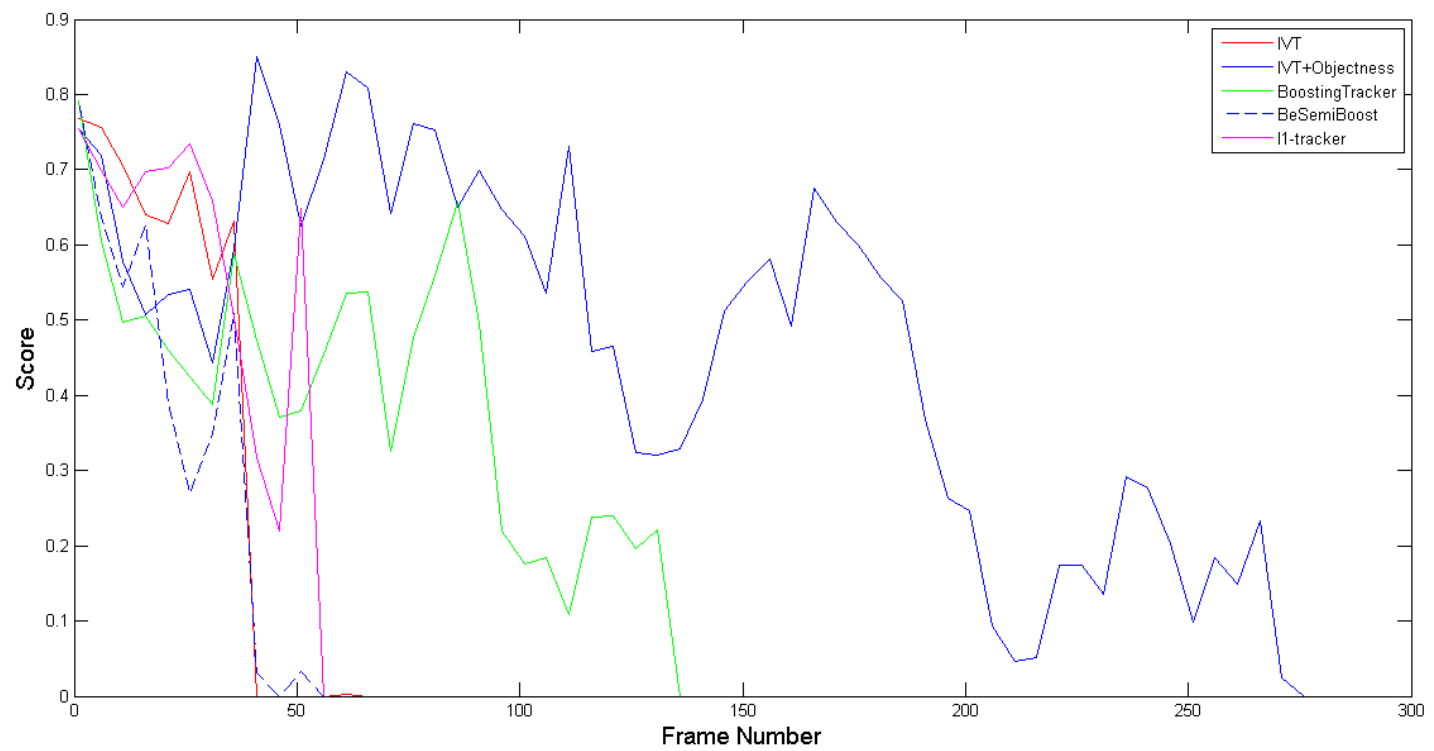

Figure 4.11 Comparison of different online trackers: Coke Can Sequence: Frame number VS Tracking score. A frame which has a score above 1/3 can be considered a success, otherwise a miss.

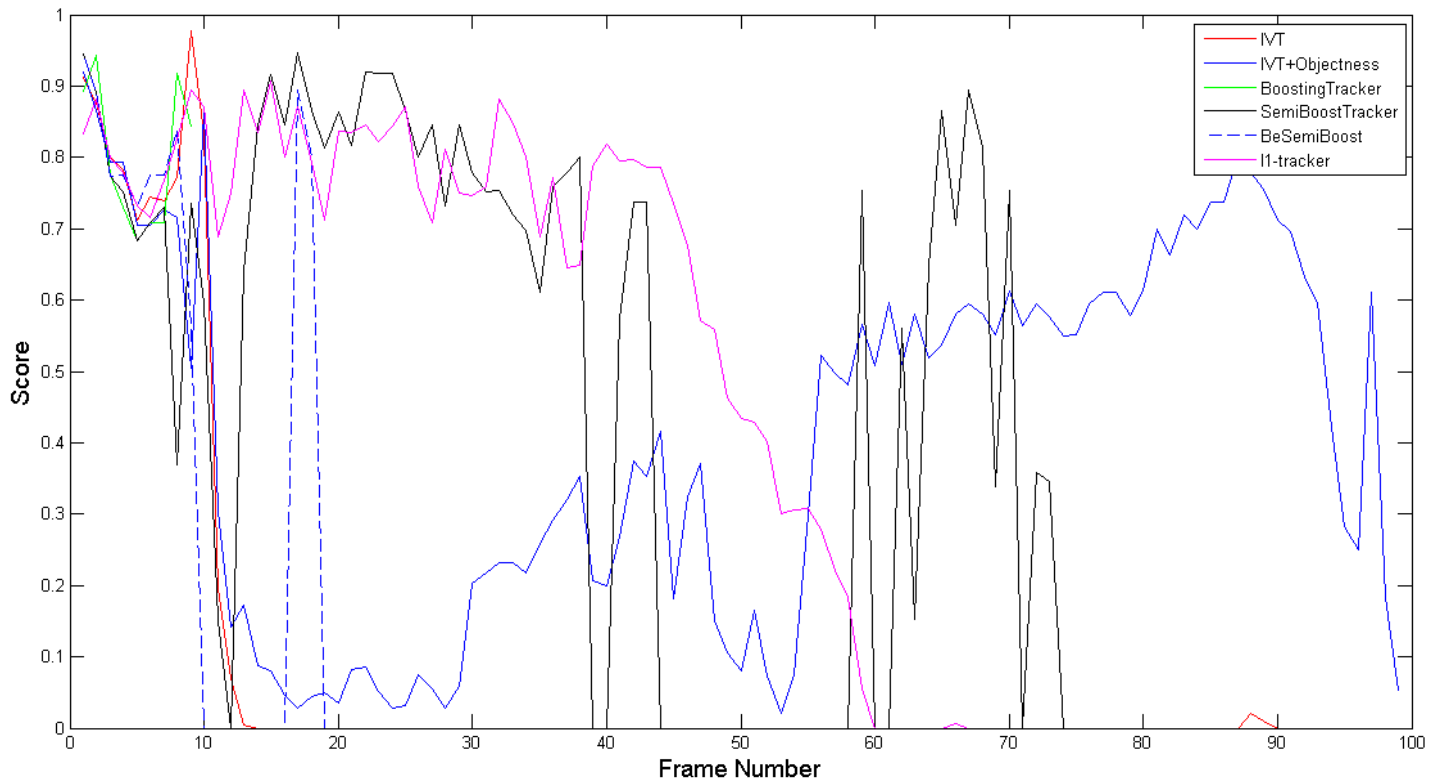

Figure 4.12 Comparison of different online trackers: Bird 2 Sequence: Frame number VS Tracking score. A frame which has a score above $1 / 3$ can be considered a success, otherwise a miss. 


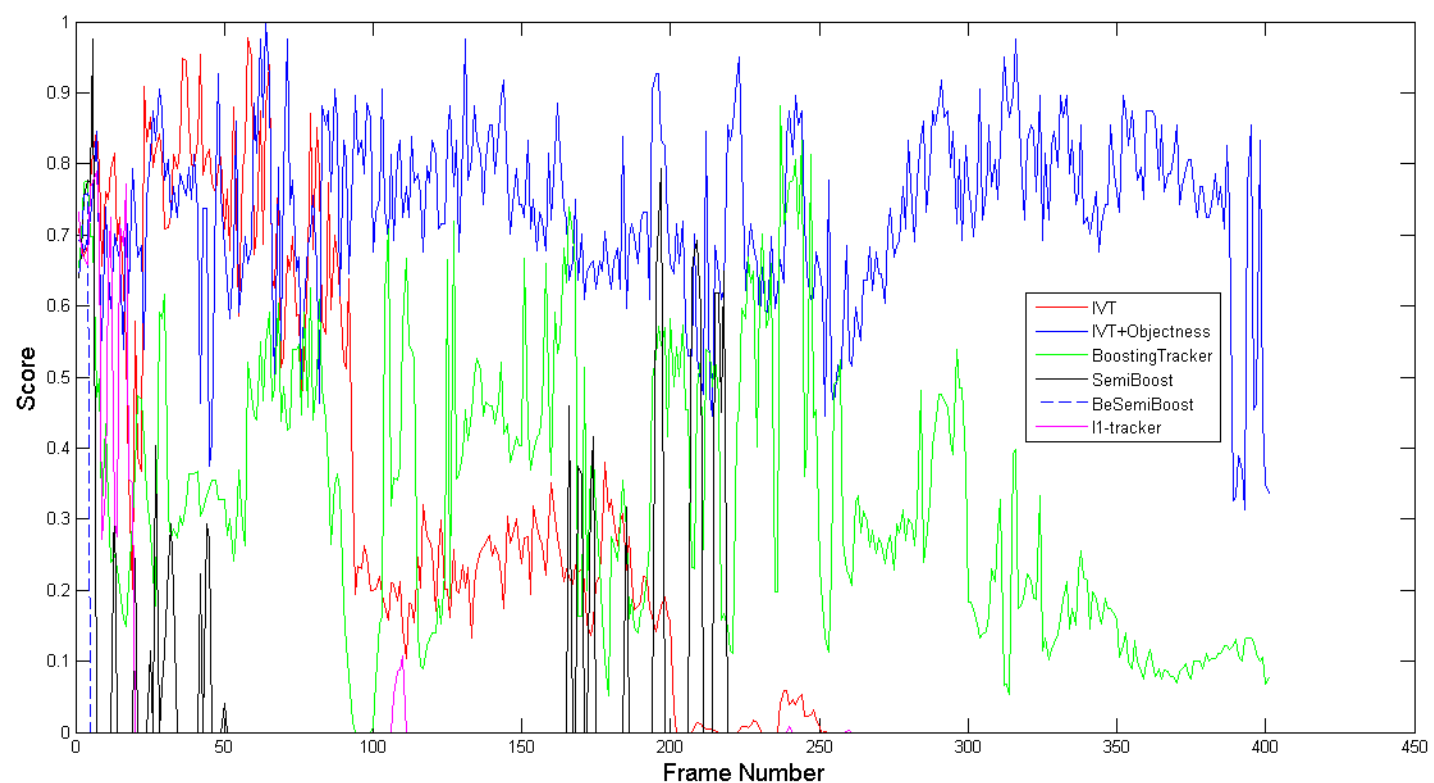

Figure 4.13 Comparison of different online trackers: Basketball Sequence: Frame number VS Tracking score. A frame which has a score above 1/3 can be considered a success, otherwise a miss.

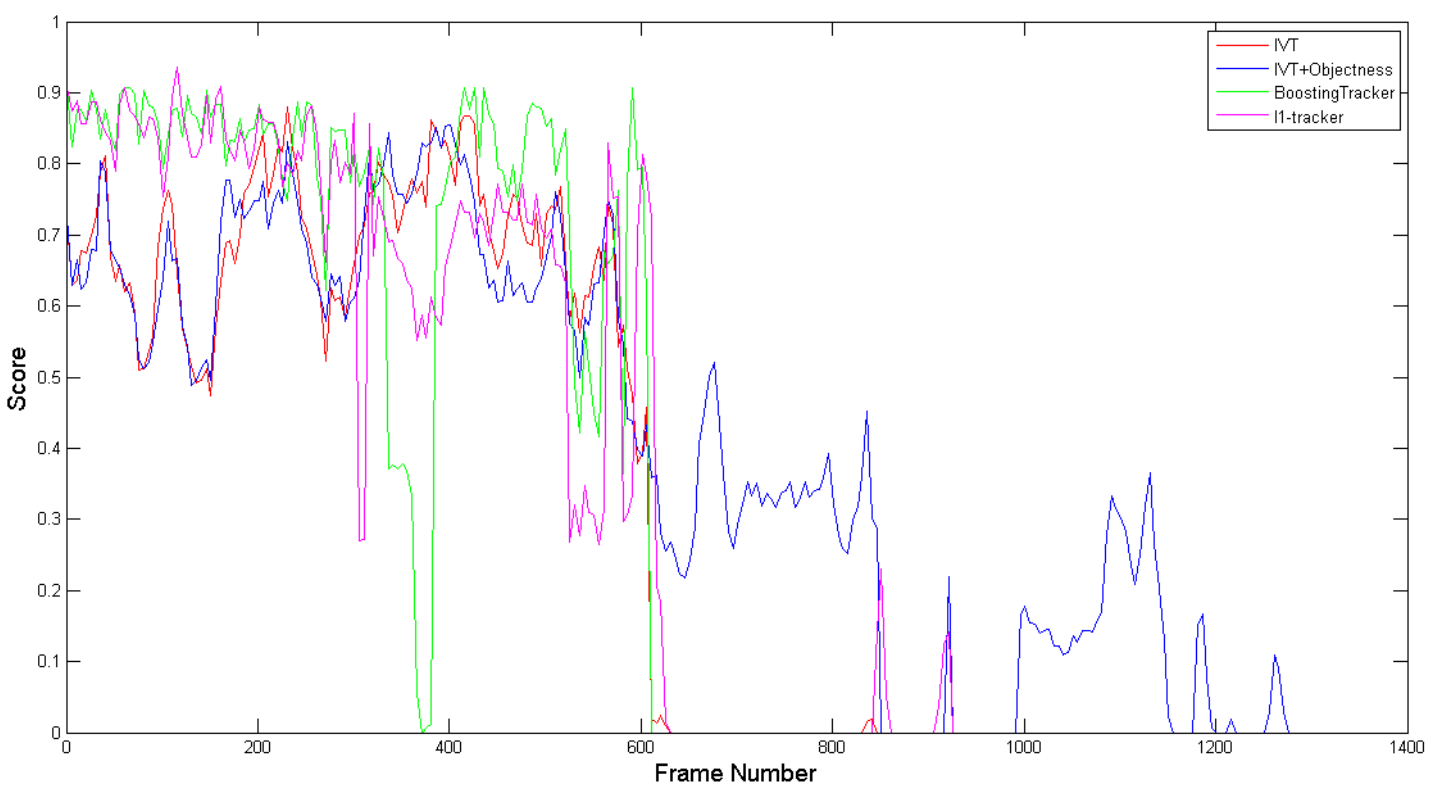

Figure 4.14 Comparison of different online trackers: Sylvester Sequence: Frame number VS Tracking score. A frame which has a score above $1 / 3$ can be considered a success, otherwise a miss. 
Table 4.3 Comparison of different online trackers: Success rates. - for a particular sequence indicates no comparison as the ground truth for these sequences is only available every 5th frame and these trackers gave intermittent detections (Hence might not yield accurate results).

\begin{tabular}{|c|c|c|c|c|c|c|}
\hline Sequence & IVT & $\begin{array}{c}\text { Proposed } \\
\text { Method }\end{array}$ & $\begin{array}{l}\text { Boost } \\
\text { Tracker }\end{array}$ & SemiBoost & BeyondSemiBoost & $\begin{array}{c}\mathrm{L} 1- \\
\text { tracker }\end{array}$ \\
\hline Coke Can & 13.70 & 61.64 & 30.82 & - & 11.99 & 15.41 \\
\hline Bird2 & 10.10 & 55.56 & 9 & 50.51 & 11.11 & 52.53 \\
\hline Basketball & 23.44 & 99.50 & 44.89 & 5.98 & 1.00 & 3.99 \\
\hline Sylvester & 45.39 & 55.06 & 43.90 & - & - & 41.67 \\
\hline Popma & 24.32 & 18.91 & 21.62 & 37.83 & 21.62 & 21.62 \\
\hline Woman & 14.11 & 14.11 & 14.11 & 31.53 & 6.30 & 15.61 \\
\hline Girlocc & 41.47 & 41.47 & 42.63 & 30.62 & 42.24 & 48.06 \\
\hline Transformer & 33.06 & 34.67 & 0.8 & 1.61 & 26.6 & 37.06 \\
\hline Bird1 & 6.12 & 6.80 & 7.48 & 19.73 & 5.44 & 9.52 \\
\hline Tiger 1 & 50.85 & 49.44 & 14.12 & - & 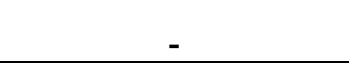 & 22.5 \\
\hline
\end{tabular}




\subsection{Failure Cases}

Here we include certain cases in which proposed method has failed and discuss possible reasons for the failure. Figure 4.15 show some of the cases in which proposed approach failed.
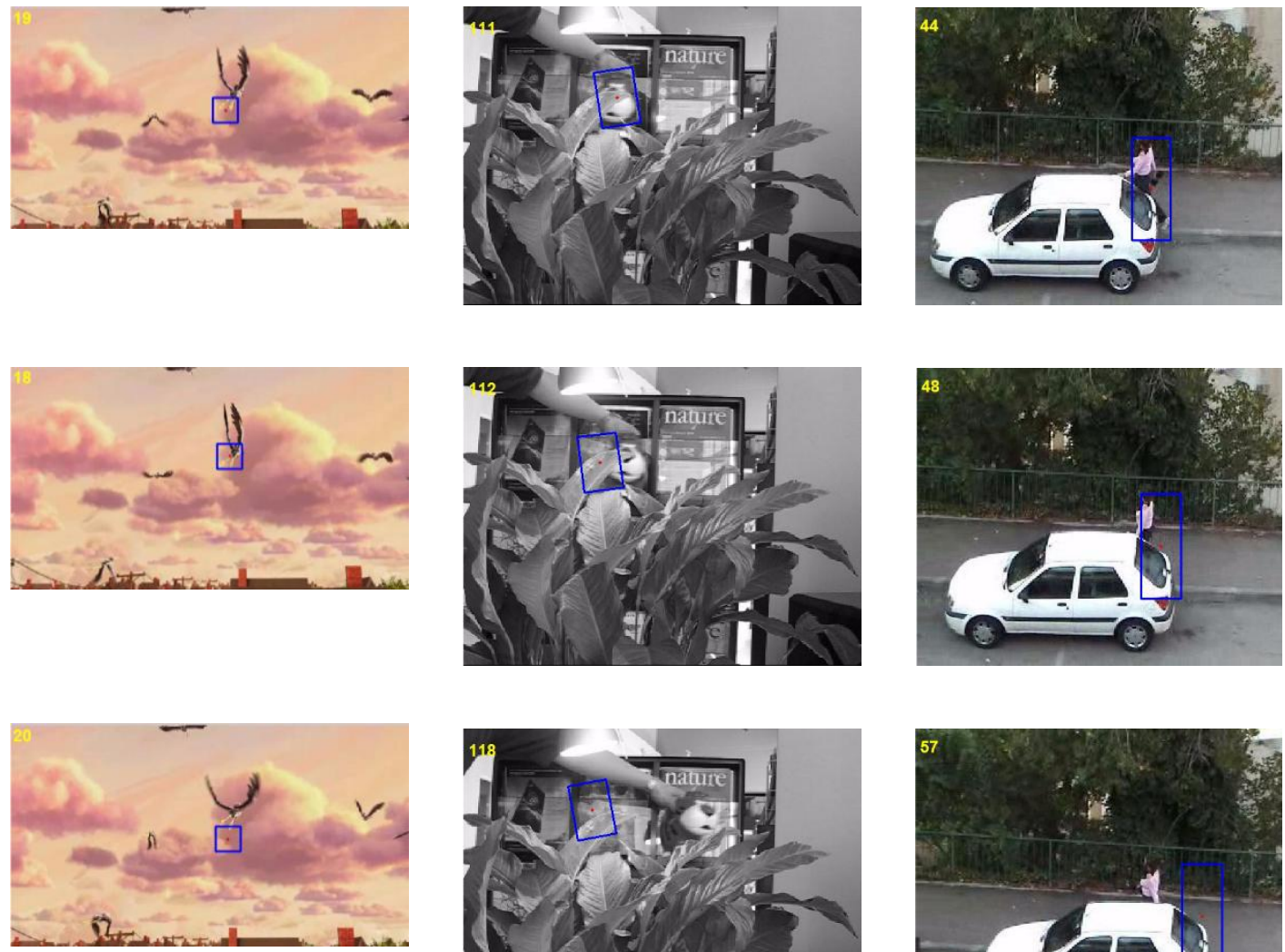

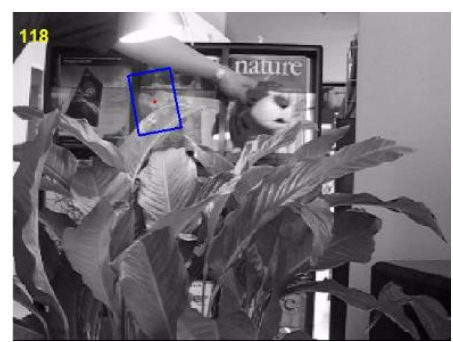

(b)

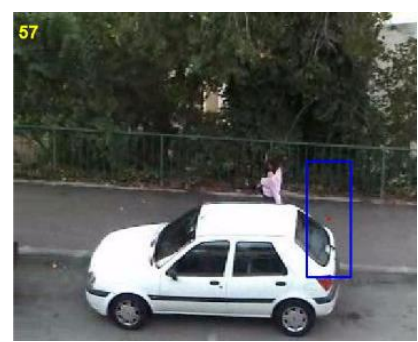

(c)

Figure 4.15 Some cases in which proposed approach failed: (a) Bird 1 sequence (b) Tiger 1 sequence (c) Woman sequence. Blue rectangle represents the output of the tracker

As shown in the Figure 4.15 (a), proposed method fails to track the bird due to smaller size of the object and fast movement of the object at the same time. As a result of small size of the target, SS cue may not perform well as superpixels are fragile for smaller objects [27].In the case of Tiger 1 sequence as depicted in 4.15 (b), there is prolonged occlusion from frames 108112 and proposed method drifts to the background(similar to the IVT). The failure may be 
attributed to the nature of the background in the sequence which might also cause difficulty in segmentation of the superpixels. In the last case listed (Figure 4.15(c)) i.e., woman sequence, proposed method again fails when woman walks behind the car making herself only partially visible. This is because both IVT and objectness might fail in the event of partial occlusion. IVT employs holistic appearance model which uses all the pixels of the object while objectness scores the windows containing complete object higher. This makes it difficult for proposed approach to handle partial occlusion. A parts based approach may be incorporated in to the appearance model of IVT to handle such cases. Also as every tracker inevitably fails in certain scenario, we propose to develop robust reinitialization schemes to reacquire the target which can improve the reliability of tracking despite different challenges. In the next and final chapter of the thesis, we discuss possible improvements and additions to our current work. 


\section{Conclusion and Future Work}

In this thesis we have studied about exploiting the objectness for online object tracking. We have incorporated objectness in to the Incremental Learning for Visual Tracking (IVT) algorithm in a principled way and developed a reinitializing mechanism to get rid of erroneous accumulations in the appearance model of the IVT. Through our extensive experiments to test the efficacy of the proposed approach we have found that objectness does help to alleviate complete drift to background problem of the IVT on certain challenging sequences.

Following are the few avenues we propose to explore as possible line of future work along the direction of current work in this thesis

- More ways to combine the two modules viz., tracking and objectness has to be further investigated. Also currently in our work, we employ two cues (Color Contrast, Superpixel Straddling) proposed in [27]. We wish to include more cues that denotes the objectness of a particular window especially cues such as Multiscale Saliency [27] which might boost the performance as the spatial information in the whole frame can be exploited via such approach.

- Thresholds currently used in the proposed algorithm are hard thresholds. We would like to make them adaptive based on the information acquired through the incoming frames of the image sequence and test the effectiveness of such approach.

- One of the primary challenges faced by state of the art tracking research is lack of proper database to evaluate tracking algorithms. Also, albeit the availability of source codes of most of the existing tracking algorithms for research purposes, comparing different trackers in fair manner is quite difficult since each tracking algorithm involves different variety of parameters and the way inputs are given and outputs are extracted differs greatly from one algorithm to another. Very recently, a code library constituting multifarious online tracking algorithms with large number of video sequences (with ground truth annotations) are formed for a benchmark study of online tracking algorithms by Wu et.al [52]. At this moment, the dataset and code library are not available due to copyright issues but once made available we wish to evaluate the proposed method on more no of video 
sequences in the dataset and compare with the different trackers to further test the effectiveness of using objectness for tracking in different scenarios.

- For applications like video based face recognition [2], accurately aligned faces from the face tracking module are critical for better recognition. We have conducted preliminary study of different ways of combining objectness with IVT for face tracking on Honda/UCSD face video database [57] [58] to see if they give better aligned faces when compared to IVT. Although an occasional qualitative improvement was observed, lack of ground truth data for this database made it difficult to test the efficacy of this approach in an accurate way. We would like to explore along this direction, and investigate the effectiveness of the idea by using a database with annotated data or by computing the recognition performance as a measure of reflectance of the tracking performance.

- Finally, no matter how robustly designed it is inevitable for any tracker to fail under a particular scenario (No tracking algorithm is ideal for all the scenarios). We are interested in exploring different ways of detecting the tracking failures and recovery methods that may augment the robustness of object tracking. 


\section{References}

[1] Q. Wang, F. Chen, W. Xu and M.-h. Yang, "Experimental Comparison of Online Object Tracking Algorithms," in SPIE, 2010.

[2] M. Kim, S. Kumar, V. Pavlovic and H. Rowley, "Face Tracking and Recognition with Visual Constraints in Real-World Videos," in CVPR, 2008.

[3] I.Haritaoglu, D.Harwood and L.Davis, "W4s: A real-time system for detecting and tracking people," in IEEE CVPR, 1998.

[4] M. L. Gorce, N.Paragios and D.Fleet, "Model-based hand tracking with texture,shading and self occlusions," in IEEE CVPR, 2008.

[5] X.Zhou, D.Comaniciu and A.Gupta, "An information fusion framework for robust shape tracking," IEEE TPAMI, vol. 27, no. 1, pp. 115-129, 2005.

[6] "CAVIAR: Context Aware Vision using Image-based Active Recognition," 1 October 2002. [Online]. Available: http://homepages.inf.ed.ac.uk/rbf/CAVIAR/caviar.htm. [Accessed 29 April 2013].

[7] B. Babenko, M.-H. Yang and S. Belongie, "Robust Object Tracking with Online Multiple Instance Learning," IEEE TPAMI, 2011.

[8] M.J.Blake and A.D.Jepson, "Robust matching and tracking of articulated objects using a view-based representation," in ECCV, 1996.

[9] M.Isard and A.Blake, "Contour tracking by stochastic propagation of conditional density," in ECCV, 1996.

[10] D.Comaniciu, V.Ramesh and P.Meer, "Kernel-based object tracking," IEEE TPAMI, vol. 25, 
no. 5, pp. 564-575, 2003.

[11] A.D.Jepson, D.J.Fleet and T.F.El-Maraghi, "Robust online appearance models for visual tracking," IEEE, vol. 25, no. 10, pp. 1296-1311, 2001.

[12] S.Avidan, "Support vector tracking," in IEEE CVPR, 2001.

[13] D. Ross, J. Lim, R.-S. Lin and M.-H. Yang, "Incremental learning for robust visual tracking," IJCV, 2007.

[14] B.Lucas and T.Kanade, "An iterative image registration technique with an application to sterio vision," in IJCAl, 1981.

[15] M. Xue and L. Haibin, "Robust visual tracking using I1-minimization," in IEEE ICCV, 2009.

[16] A.Azarbayejani and A.Pentland, "Recursive estimation of motion,structure, and focal length," IEEE TPAMI, vol. 17, no. 6, pp. 562-575, 1995.

[17] D.A.Klein, D.Schulz, S.Frintrop and A.B.Cremers, "Adaptive Real Time Video Tracking for Arbitrary Objects," in IEEE Intelligent Robots and Systems, 2010.

[18] J. Kwon and K. M. Lee, "Visual Tracking Decomposition,," in IEEE CVPR, 2010.

[19] A. Adam, E. Rivlin and I. Shimshoni, " Robust Fragments-based Tracking using the Integral Histogram," in IEEE CVPR, 2006.

[20] H. Grabner, M. Grabner and H. Bischof, "Real-time Tracking via On-line Boosting," in IEEE BMVC, 2006.

[21] H. Grabner, C. Leistner and H. Bischof, "Semi-supervised On-line Boosting for Robust Tracking," in ECCV, 2008.

[22] S. Stalder, H. Grabner and L. V. Gool, "Beyond Semi-Supervised Tracking: Tracking Should Be as Simple as Detection, but not Simpler than Recognition," in IEEE ICCV, 2009.

[23] R. Collins and Y. Liu, "On-line selection of discriminative tracking features," in ICCV, 2003. 
[24] Z. Kalal, K. Mikolajczyk and J. Matas, "Tracking-Learning-Detection," IEEE TPAMI, vol. 34, no. 7, pp. $1409-1422$, July 2012.

[25] T. B. Dinh, N. Vo and G. Medioni, " Context Tracker: Exploring Supporters and Distracters in Unconstrained Environments," in CVPR, 2011.

[26] K. Cannons, J. Gryn and R. Wildes, " Visual Tracking using a Pixelwise Spatiotemporal Oriented Energy Representation," in ECCV, 2010.

[27] B. Alexe, T. Deselares and V. Ferrari, "Measuring the objectness of image windows," TPAMI, 2012.

[28] B. Alexe, T. Deselaers and V. Ferrari, "What is an Object?," in CVPR, 2010.

[29] S. Stadler, H. Grabner and L. Van Gool, "Dynamic Objectness for Adaptive Tracking," in ACCV, 2012.

[30] A. L. a. M. Lindenbaum., "Sequential Karhunen-Loeve basis extraction and its application to images," IEEE TPAMI, vol. 9, no. 8, pp. 1371-1374, 2000.

[31] H. Golub and C. F. V. Loan, Matrix computations, The Johns Hopkins University Press, 1996.

[32] P. Hall, D. Marshall and R. Martin, "Incremental eigenanalysis for classification," in BMVC, 1998.

[33] M. Brand, "Incremental singular value decomposition of uncertain data with missing value," in ECCV, 2002.

[34] M. E. Tipping and C. M. Bishop, "Probabilistic principal component analysis," Journal of the Royal Statistical Society, vol. 61, no. 3, pp. 611-622, 1999.

[35] B. Babenko, M.-H. Yang and S. Belongie, "Visual Tracking with Online Multiple Instance Learning," in CVPR, 2009. 
[36] S. Wang, H. Lu, F. Yang and M.-H. Yang, "Superpixel Tracking," in ICCV, 2011.

[37] B. Alexe, T. Deselaers and V. Ferrari, "What is an object ?," in CVPR, 2010.

[38] N. Bruce and J. Tsotsos, " Saliency based on information maximization," in NIPS, 2005.

[39] D. Gao and N. Vasconcelos, " Bottom-up saliency is a discriminant process," in ICCV, 2007.

[40] X. Hou and L. Zhang., "Saliency detection: A spectral residual approach," in CVPR, 2007.

[41] L. Itti, C. Koch and E. Niebur., "A model of saliency-based visual attention for rapid scene analysis," IEEE TPAMI, vol. 20, no. 11, pp. 1254-1259, 1998.

[42] T. Liu, J. Sun, N. Zheng, X. Tang and H. Shum., "Learning to detect a salient object," in CVPR, 2007.

[43] Y. F. Ma and H. J. Zhang, "Contrast-based image attention analysis by using fuzzy growing," in ICMM, 2003.

[44] P. F. Felzenszwalb and D. P. Huttenlocher, " Efficient graph-based image segmentation," IJCV, vol. 59, no. 2, p. 167-181, September,2004.

[45] B. C. Russell, A. A. Efros, J. Sivic, W. T. Freeman and A. Zisserman, "Using multiple segmentations to discover objects and their extent in image collections," in CVPR, 2006.

[46] X. Jia, H. Lu and M.-H. Yang, "Visual Tracking via Adaptive Structural Local Sparse Appearance Model," in CVPR, 2012.

[47] K. Brocklehurst, "Particle Filtering for Face Tracking," Fall 2010. [Online]. Available: http://vision.cse.psu.edu/people/kyleB/faceTracker/index.shtml. [Accessed 2 May 2013].

[48] Z. Kalal, K. Mikolajczyk and J. Matas, "Forward-Backward Error: Automatic Detection of Tracking Failures," in ICPR, 2010.

[49] W. Hao, A. C. Sankaranarayanan and R. Chellappa, "Online Empirical Evaluation of 
Tracking Algorithms," TPAMI, vol. 32, no. 8, pp. 1443-1458, 2010.

[50] R. B. Fisher, "The PETS04 Surveillance Ground-Truth Data Sets," in PETS, 2004.

[51] R. T. Collins, X. Zhou and S. K. Teh, "An Open Source Tracking Testbed and Evaluation Web Site," in PETS, 2005.

[52] Y. Wu, J. Lim and M.-H. Yang, "Online Object Tracking: A Benchmark," in CVPR, 2013.

[53] P. Chockalingam, N. Pradeep and S. Birchfield, " Adaptive Fragments-Based Tracking of Non-Rigid Objects Using Level Sets," in ICCV, 2009.

[54] J. A.D, F. D.J and E.-M. T., "Robust online appearance models for visual tracking," IEEE TPAMI, vol. 25, no. 10, pp. 1296-1311, 2003.

[55] S. M. S. Nejhum, J. Ho and M.-H. Yang, "Visual Tracking with Histograms and Articulating Blocks," in CVPR, 2008.

[56] J. Kwon and K. M. Lee, "Tracking of a Non-Rigid Object via Patch-based Dynamic Appearance Modeling and Adaptive Basin Hopping Monte Carlo Sampling," in CVPR, 2009.

[57] K.-C. Lee, J. Ho, M.-H. Yang and D. Kriegman, "Visual Tracking and Recognition Using Probabilistic Appearance Manifolds," in CVIU, 2005.

K.-C. Lee and D. Kriegman, "Online Learning of Probabilistic Appearance Manifolds for 58] Video-based Recognition and Tracking," in CVPR, 2005. 